\title{
Proof of NRQCD factorization at all orders in the coupling constant in heavy quarkonium production
}

\author{
Gouranga C. Nayak ${ }^{\mathrm{a}}$ \\ 665 East Pine Street, Long Beach, NY 11561, USA
}

Received: 15 December 2015 / Accepted: 1 August 2016 / Published online: 11 August 2016

(C) The Author(s) 2016. This article is published with open access at Springerlink.com

\begin{abstract}
Recently the proof of the factorization in heavy quarkonium production in the NRQCD color octet mechanism is given at next-to-next-to-leading order (NNLO) in the coupling constant by using diagrammatic method of QCD. In this paper we prove factorization in heavy quarkonium production in the NRQCD color octet mechanism at all orders in the coupling constant by using the path integral method of QCD. Our proof is valid to all powers in the relative velocity of the heavy quark. We find that the gauge invariance and the factorization at all orders in the coupling constant require gauge-completed non-perturbative NRQCD matrix elements that were introduced previously to prove factorization at NNLO.
\end{abstract}

\section{Introduction}

In the last two decades, the NRQCD color octet mechanism [1] for heavy quarkonium production has been very successful in explaining experimental data at high energy colliders such as at Tevatron [2-10] and at LHC [11-28]. In its original formulation [1] the proof of the factorization in heavy quarkonium production in the NRQCD color octet mechanism was lacking. The proof of the factorization is an essential requirement to study heavy quarkonium production at high energy colliders. Factorization refers to separation of short-distance effects from long-distance effects in quantum field theory.

Recently the proof of the factorization in heavy quarkonium production in the NRQCD color octet mechanism is given at next-to-next to leading order (NLLO) in the coupling constant by using diagrammatic method of QCD [2931]. However, the proof of factorization in heavy quarkonium production in the NRQCD color octet mechanism at all orders in the coupling constant is still missing. In this paper we will prove factorization in heavy quarkonium production in the

\footnotetext{
a e-mail: nayakbp@aol.com
}

NRQCD color octet mechanism at all orders in the coupling constant by using the path integral method of QCD.

The typical non-perturbative NRQCD matrix element in heavy quarkonium production is given by [1]

$$
\left\langle 0\left|\mathcal{O}_{n}\right| 0\right\rangle=\left\langle 0\left|\chi^{\dagger}(0) K_{n} \xi(0)\left(a_{H}^{\dagger} a_{H}\right) \xi^{\dagger}(0) K_{n}^{\prime} \chi(0)\right| 0\right\rangle
$$

where $\xi$ is the two component Dirac spinor field that annihilates a heavy quark, $\chi$ is the two component Dirac spinor field that creates a heavy quark, $a_{H}^{\dagger}$ is the operator that creates the heavy quarkonium $H$ in the out state. The factors $K_{n}$ and $K_{n}^{\prime}$ are products of a color matrix (either a unit matrix or $T^{a}$ ), a spin matrix (either a unit matrix or $\sigma^{i}$ ), and a polynomial of the covariant derivative $D$. The color and spin indices on the fields $\chi$ and $\xi$ have been suppressed.

The production cross section for heavy quarkonium $H$ at transverse momentum $P_{T}$ in NRQCD factorizes into a sum of perturbative functions times universal matrix elements,

$\mathrm{d} \sigma_{A+B \rightarrow H+X\left(P_{T}\right)}=\sum_{n} \mathrm{~d} \hat{\sigma}_{A+B \rightarrow Q \bar{Q}[n]+X\left(P_{T}\right)}\left\langle\mathcal{O}_{n}\right\rangle$

where each NRQCD non-perturbative matrix element $\left\langle\mathcal{O}_{n}\right\rangle$ represents the probability of a heavy quark-antiquark pair in state $[n]$, such as a color singlet or a color octet etc., to produce the heavy quarkonium state $H$.

The fragmentation function for parton $i$ to evolve into a heavy quarkonium at large $P_{T}$ is factorized according to [32]

$D_{H / i}\left(z, m_{c}, \mu\right)=\sum_{n} d_{i \rightarrow Q \bar{Q}[n]}\left(z, m_{c}, \mu\right)\left\langle\mathcal{O}_{n}\right\rangle$

in terms of the same NRQCD non-perturbative matrix elements, along with perturbative functions $d_{i \rightarrow Q \bar{Q}[n]}\left(z, m_{c}, \mu\right)$ that describe the evolution of an off-shell parton into a heavy quark-antiquark pair in state $[n]$, such as a color singlet or a color octet etc.

In a first glance it can easily be seen that the nonperturbative NRQCD matrix element in Eq. (1) is not gauge invariant unless it is a color singlet $\mathrm{S}$-wave non-perturbative matrix element. Hence one expects that any non-canceling 
infrared divergences in the perturbative Feynman diagrams of heavy quark-antiquark production with short-distance coefficient cannot be factorized into the definition of the nonperturbative NRQCD matrix element in Eq. (1) in the study of heavy quarkonium production at high energy colliders in the NRQCD color octet mechanism.

This is explicitly shown in [29-31] where the NNLO coupling constant calculation shows that the above nonperturbative NRQCD matrix element in Eq. (1) is not consistent with factorization of infrared divergences unless it is a color singlet $\mathrm{S}$-wave non-perturbative matrix element. By using the calculation at NNLO in the coupling constant and to all powers in the relative velocity of the heavy quark it was shown in [29-31] that the octet S-wave non-perturbative NRQCD matrix element which is gauge invariant and is consistent with the factorization of infrared divergences is given by

$$
\begin{aligned}
\left\langle 0\left|\mathcal{O}_{n}\right| 0\right\rangle= & \langle 0| \chi^{\dagger}(0) K_{n, e} \xi(0) \Phi_{l}^{(A) \dagger}(0)_{e b} \\
& \times\left(a_{H}^{\dagger} a_{H}\right) \Phi_{l}^{(A)}(0)_{b a} \xi^{\dagger}(0) K_{n, a}^{\prime} \chi(0)|0\rangle
\end{aligned}
$$

where

$$
\begin{aligned}
\Phi_{l}^{(A)}(0)= & \mathcal{P} \exp \left[-i g T^{(A) c} \int_{0}^{\infty} \mathrm{d} \lambda l \cdot A^{c}(l \lambda)\right], \\
& \left(T^{(A) c}\right)_{a b}=-i f^{a b c}
\end{aligned}
$$

is the gauge link or the non-abelian phase in the adjoint representation of $\mathrm{SU}(3), A^{\mu a}(x)$ is the gluon field, $\mathcal{P}$ is the path ordering and $l^{\mu}$ is the light-like four-velocity.

Note that a necessary condition for NRQCD factorization is that the long-distance behavior of the non-perturbative NRQCD matrix element must be independent of the lightlike vector $l^{\mu}$. Such a dependence would be inconsistent with NRQCD factorization because the infrared divergences of $\left\langle\mathcal{O}_{n}\right\rangle$ must match those of the cross sections, in which there is no information on $l^{\mu}$. In [29-31] we have verified the $l^{\mu}$ independence of the infrared pole at NNLO in the coupling constant and to all powers in the relative velocity of the heavy quark.

Since the NRQCD matrix element in Eq. (1) is a nonperturbative quantity in QCD it cannot be calculated by using perturbative QCD methods. It is well known that a nonperturbative function cannot be studied by using perturbative methods, no matter how many orders of perturbation theory are used. Hence the path integral formulation (as opposed to diagrammatic method using perturbation theory) is necessary to study the properties of non-perturbative quantities in QCD at all orders in the coupling constant. The only path integral formulation to study factorization of soft and collinear divergences at all orders in the coupling constant in quantum field theory is given by Tucci in [33], which is exact for QED but is not exact for QCD. We have extended this path integral approach to QCD to prove factorization in QCD at all orders in the coupling constant in [34]. In this paper we will extend this path integral approach to prove NRQCD factorization at all orders in the coupling constant in heavy quarkonium production. We will prove that the long-distance behavior of the non-perturbative NRQCD matrix element is independent of the light-like vector $l^{\mu}$ at all orders in the coupling constant.

The paper is organized as follows. In Sect. 2 we briefly describe the lagrangian density in NRQCD and in QCD. In Sect. 3 we discuss infrared divergences in NRQCD and in QCD. In Sect. 4 we include the heavy quark in the path integral formulation of QCD. In Sect. 5 we describe infrared divergences in NRQCD and the light-like Wilson line in QCD. In Sect. 6 we show that the eikonal current of the light-like charge generates pure gauge field in quantum field theory. In Sect. 7 we show how the pure gauge field in quantum field theory can be used to describe soft (infrared) divergences. In Sect. 8 we study the heavy quark-antiquark nonperturbative matrix element in the presence of a light-like Wilson line in QCD. In Sect. 9 we prove factorization in heavy quarkonium production in the NRQCD color octet mechanism at all orders in the coupling constant and to all powers in the relative velocity of the heavy quark. In Sect. 10 we show that the factorization theorem is a key ingredient in calculation of NRQCD heavy quarkonium production cross section at collider experiments. Section 11 contains conclusions.

\section{Lagrangian density in NRQCD and in QCD}

The lagrangian density in QCD including heavy quarks is given by [35]

$$
\begin{aligned}
\mathcal{L}_{\mathrm{QCD}}= & -\frac{1}{4} F_{\mu \nu}^{a} F^{\mu \nu a}+\sum_{l=1}^{3} \bar{\psi}_{l}\left[\gamma^{\mu} D_{\mu}-m_{l}\right] \psi_{l} \\
& +\bar{\Psi}\left[\gamma^{\mu} D_{\mu}-M\right] \Psi
\end{aligned}
$$

where $F^{\mu \nu a}$ is the full non-abelian gluon field tensor, $\psi_{l}$ is the Dirac field of the light quark $(l=u, d, s), \Psi$ is the Dirac field of the heavy quark, $D^{\mu}$ is the covariant derivative, $\gamma^{\mu}$ is the Dirac matrix, $m_{l}$ is the mass of the light quark and $M$ is the mass of the heavy quark.

In NRQCD an ultraviolet cutoff $\Lambda \sim M$ is introduced. The lagrangian density in NRQCD is given by [1]

$\mathcal{L}_{\mathrm{NRQCD}}=\mathcal{L}_{\text {light }}+\mathcal{L}_{\text {heavy }}+\delta \mathcal{L}$

where

$\mathcal{L}_{\text {light }}=-\frac{1}{4} F_{\mu \nu}^{a} F^{\mu \nu a}+\sum_{l=1}^{3} \bar{\psi}_{l}\left[D-m_{l}\right] \psi_{l}$, 
$\mathcal{L}_{\text {heavy }}=\xi^{\dagger}\left[i D_{t}+\frac{\mathbf{D}^{2}}{2 M}\right] \xi+\chi^{\dagger}\left[i D_{t}-\frac{\mathbf{D}^{2}}{2 M}\right] \chi$,

and

$$
\begin{aligned}
\delta \mathcal{L}= & \frac{c_{1}}{8 M^{3}}\left[\xi^{\dagger}\left(\mathbf{D}^{2}\right)^{2} \xi-\chi^{\dagger}\left(\mathbf{D}^{2}\right)^{2} \chi\right] \\
& +\frac{c_{2}}{8 M^{2}}\left[\xi^{\dagger}(\mathbf{D} \cdot g \mathbf{E}-g \mathbf{E} \cdot \mathbf{D}) \xi\right. \\
& \left.-\chi^{\dagger}(\mathbf{D} \cdot g \mathbf{E}-g \mathbf{E} \cdot \mathbf{D}) \chi\right] \\
& +\frac{c_{3}}{8 M^{2}}\left[\xi^{\dagger}(i \mathbf{D} \times g \mathbf{E}-g \mathbf{E} \times i \mathbf{D})\right. \\
& \left.+\sigma \xi-\chi^{\dagger}(i \mathbf{D} \times g \mathbf{E}-g \mathbf{E} \times i \mathbf{D}) \cdot \sigma \chi\right] \\
& +\frac{c_{4}}{2 M}\left[\xi^{\dagger} g \mathbf{B} \cdot \sigma \xi-\chi^{\dagger} g \mathbf{B} \cdot \sigma \chi\right]+\cdots
\end{aligned}
$$

where $D_{t}$ and $\mathbf{D}$ are the time and space components of the covariant derivative $D^{\mu}$ and $\mathbf{E}$ and $\mathbf{B}$ are electric and magnetic components of the gluon field tensor and $\sigma$ is the Pauli spin matrix. The dimensionless coefficients $c_{1}, c_{2}, c_{3}, c_{4}$, etc. in Eq. (10) are obtained by matching NRQCD with QCD [1].

\section{Infrared behavior in NRQCD and in QCD}

Note that in order for the factorization formula to hold in Eqs. (2) and (3) the perturbative functions have to be infraredsafe by definition because the infrared limit corresponds to long-distance regime [1]. However, as found in $[29,30]$ the NNLO infrared pole contribution to order $\vec{v}^{2}$ is given by

$\Sigma(P, v, l)=\alpha_{s}^{2} \frac{1}{3 \epsilon} \frac{\vec{v}^{2}}{4}$

which is not zero where $\vec{v}$ is the relative velocity of the heavy quark-antiquark pair. Equation (11) is in the rest frame of the heavy quarkonium $(\vec{P}=0)$ where $P^{\mu}$ is the fourmomentum of the heavy quarkniuom and $l^{\mu}$ is the fourvelocity of the light-like Wilson line which is fixed to be $l^{\mu}=\delta^{\mu-}$ along the minus light cone direction in [2931]. The presence of a non-zero infrared pole in Eq. (11) implies that infrared poles will appear in perturbative functions at NNLO and beyond when the factorization is carried out with octet non-perturbative NRQCD matrix element $\left\langle\chi^{\dagger} K_{n} \xi\left(a_{H}^{\dagger} a_{H}\right) \xi^{\dagger} K_{n}^{\prime} \chi\right\rangle$ in the conventional manner as given by Eq. (1) in Eqs. (2) and (3). On the other hand, when defined according to its gauge-completed form as given by Eq. (4) each octet non-perturbative NRQCD matrix element itself generates precisely the same pole terms given in Eq. (11) above. This conclusion is valid to all powers in $v$ at NNLO in the coupling constant [31]. Thus NRQCD can accommodate these corrections. Hence our main aim in this paper is to prove that Eq. (4) is valid at all orders in the coupling constant.

Note that in NRQCD an ultraviolet cutoff $\Lambda \sim M$ is introduced [1]. Hence the ultraviolet (UV) behavior of QCD and NRQCD differ. However, the infrared (IR) behavior of QCD and NRQCD remains same [36]. Hence the infrared behavior in NRQCD can be obtained by studying the corresponding infrared behavior in QCD. Since the matrix element of the type $\left\langle\chi^{\dagger} K_{n} \xi\left(a_{H}^{\dagger} a_{H}\right) \xi^{\dagger} K_{n}^{\prime} \chi\right\rangle$ is the non-perturbative NRQCD matrix element, it is natural to study its infrared behavior at all orders in the coupling constant by using the path integral method. Hence we will use the path integral method of QCD in this paper.

\section{Heavy quarks and the path integral formulation of QCD}

The generating functional in QCD including the heavy quark is given by $[35,37]$

$$
\begin{aligned}
& Z\left[J, \eta_{u}, \bar{\eta}_{u}, \eta_{d}, \bar{\eta}_{d}, \eta_{s}, \bar{\eta}_{s}, \eta_{h}, \bar{\eta}_{h}\right] \\
& =\int[\mathrm{d} Q]\left[\mathrm{d} \bar{\psi}_{1}\right]\left[\mathrm{d} \psi_{1}\right]\left[\mathrm{d} \bar{\psi}_{2}\right]\left[\mathrm{d} \psi_{2}\right]\left[\mathrm{d} \bar{\psi}_{3}\right]\left[\mathrm{d} \psi_{3}\right][\mathrm{d} \bar{\Psi}][\mathrm{d} \Psi] \\
& \quad \times \operatorname{det}\left(\frac{\delta\left(\partial_{\mu} Q^{\mu a}\right)}{\delta \omega^{b}}\right) \\
& \quad \times e^{i} \int \mathrm{d}^{4} x\left[-\frac{1}{4} F_{\mu \nu}^{a 2}[Q]-\frac{1}{2 \alpha}\left(\partial_{\mu} Q^{\mu a}\right)^{2}+J \cdot Q\right. \\
& \quad+\sum_{l=1}^{3}\left[\bar{\psi}_{l}\left[i \gamma^{\mu} \partial_{\mu}-m_{l}+g T^{a} \gamma^{\mu} Q_{\mu}^{a}\right] \psi_{l}\right. \\
& \left.\quad+\bar{\eta}_{l} \psi_{l}+\bar{\psi}_{l} \eta_{l}\right]+\bar{\Psi}\left[i \gamma^{\mu} \partial_{\mu}-M\right. \\
& \left.\left.\quad+g T^{a} \gamma^{\mu} Q_{\mu}^{a}\right] \Psi+\bar{\eta}_{h} \Psi+\bar{\Psi} \eta_{h}\right]
\end{aligned}
$$

where $Q^{\mu a}$ is the quantum gluon field, the symbols $l=$ $1,2,3=u, d, s$ stand for three light quarks $u, d, s$, and the symbol $h$ stands for heavy quark and

$F_{\mu \nu}^{a}[Q]=\partial_{\mu} Q_{\nu}^{a}(x)-\partial_{\nu} Q_{\mu}^{a}(x)+g f^{a b c} Q_{\mu}^{b}(x) Q_{\nu}^{c}(x)$,

$F_{\mu \nu}^{a 2}[Q]=F_{\mu \nu}^{a}[Q] F^{\mu \nu a}[Q]$.

In Eq. (12) the $\bar{\eta}_{u}, \bar{\eta}_{d}, \bar{\eta}_{s}$ are external sources for $u, d, s$ quark fields, respectively, and $\bar{\eta}_{h}$ is the external source for the heavy quark field and the term $\frac{\delta\left(\partial_{\mu} Q^{\mu a}\right)}{\delta \omega^{b}}$ is the derivative of the gauge fixing term under an infinitesimal gauge transformation $[35,37]$

$\delta Q^{\mu a}=g f^{a b c} Q^{\mu b} \omega^{c}+\partial^{\mu} \omega^{a}$.

Note that the determinant $\operatorname{det}\left(\frac{\delta\left(\partial_{\mu} Q^{\mu a}\right)}{\delta \omega^{b}}\right)$ in Eq. (12) can be expressed in terms of path integration over the ghost fields [35]. However, we will directly work with the determinant $\operatorname{det}\left(\frac{\delta\left(\partial_{\mu} Q^{\mu a}\right)}{\delta \omega^{b}}\right)$ in Eq. (12).

For the heavy quark Dirac field $\Psi(x)$, the non-perturbative matrix element of the type $\left\langle 0\left|\bar{\Psi}(x) O_{n} \Psi(x) \bar{\Psi}\left(x^{\prime}\right) O_{n}^{\prime} \Psi\left(x^{\prime}\right)\right| 0\right\rangle$ in $\mathrm{QCD}$ is given by [33] 


$$
\begin{aligned}
& \left\langle 0\left|\bar{\Psi}(x) O_{n} \Psi(x) \bar{\Psi}\left(x^{\prime}\right) O_{n}^{\prime} \Psi\left(x^{\prime}\right)\right| 0\right\rangle \\
& =\frac{\delta}{\delta \eta_{h}(x)} O_{n} \frac{\delta}{\delta \bar{\eta}_{h}(x)} \frac{\delta}{\delta \eta_{h}\left(x^{\prime}\right)} O_{n}^{\prime} \frac{\delta}{\delta \bar{\eta}_{h}\left(x^{\prime}\right)} \\
& \quad \times Z\left[J, \eta_{u}, \bar{\eta}_{u}, \eta_{d}, \bar{\eta}_{d}, \eta_{s}, \bar{\eta}_{s}, \eta_{h}, \bar{\eta}_{h}\right] \\
& \quad \times\left.\right|_{J=\eta_{u}=\bar{\eta}_{u}=\eta_{d}=\bar{\eta}_{d}=\eta_{s}=\bar{\eta}_{s}=\eta_{h}=\eta_{h}=0}
\end{aligned}
$$

if the factors $O_{n}$ and $O_{n}^{\prime}$ are independent of quantum fields where the suppression of the normalization factor $Z[0]$ is understood as it will cancel in the final result (see Eq. (133)).

\section{Infrared divergences in NRQCD and light-like Wilson line in QCD}

The gauge transformation of the quark field in QCD is given by

$\psi^{\prime}(x)=e^{i g T^{a} \omega^{a}(x)} \psi(x)$.

Hence one finds that the issue of gauge invariance and factorization of infrared divergences in QCD can be simultaneously explained if $\omega^{a}(x)$ can be related to the gluon field $A^{\mu a}(x)$.

Before proceeding to the issue of gauge invariance and the factorization of infrared divergences in QCD let us first discuss the corresponding situation in QED. The gauge transformation of the Dirac field of the electron in QED is given by

$\psi^{\prime}(x)=e^{i e \omega(x)} \psi(x)$.

Hence we can expect to address the issue of gauge invariance and factorization of infrared divergences in QED simultaneously if we can relate the $\omega(x)$ to the photon field $A^{\mu}(x)$.

In QED the infrared (or soft) divergence arises only from the emission of a photon for which all components of the four-momentum are small. The eikonal propagator times the eikonal vertex for a soft photon with momentum $k$ interacting with a light-like electron moving with four-momentum $p^{\mu}$ is given by $[33,34,38-47]$

$e \frac{p^{\mu}}{p \cdot k+i \epsilon}=e \frac{l^{\mu}}{l \cdot k+i \epsilon}$

where $l^{\mu}$ is the four-velocity of the light-like electron. Note that when we say the "light-like electron" we mean the electron that is traveling at its highest speed which is arbitrarily close to the speed of light $(|\vec{l}| \sim 1)$ as it cannot travel exactly at the speed of light $(|\vec{l}|=1)$ because it has finite mass even if the mass of the electron is very small. From Eq. (18) we find

$$
\begin{aligned}
& e \int \frac{\mathrm{d}^{4} k}{(2 \pi)^{4}} \frac{l \cdot A(k)}{l \cdot k+i \epsilon}=-e i \int_{0}^{\infty} \mathrm{d} \lambda \\
& \quad \times \int \frac{\mathrm{d}^{4} k}{(2 \pi)^{4}} e^{i l \cdot k \lambda} l \cdot A(k)=i e \int_{0}^{\infty} \mathrm{d} \lambda l \cdot A(l \lambda)
\end{aligned}
$$

where the photon field $A^{\mu}(x)$ and its Fourier transform $A^{\mu}(k)$ are related by

$A^{\mu}(x)=\int \frac{\mathrm{d}^{4} k}{(2 \pi)^{4}} A^{\mu}(k) e^{i k \cdot x}$.

From Eq. (19) we find

ie $\int_{0}^{\infty} \mathrm{d} \lambda l \cdot A(l \lambda)=i \int \mathrm{d}^{4} x J^{\mu}(x) A_{\mu}(x)$

where the eikonal current density $J^{\mu}(x)$ for the light-like charge $e$ is given by

$J^{\mu}(x)=e l^{\mu} \int \mathrm{d} \lambda \delta^{(4)}(x-l \lambda)$.

Now consider the corresponding Feynman diagram for the infrared divergences in QED due to the exchange of two soft photons of four-momenta $k_{1}^{\mu}$ and $k_{2}^{\mu}$. The corresponding eikonal contribution due to the exchange of two soft photons is analogously given by

$$
\begin{aligned}
e^{2} & \int \frac{\mathrm{d}^{4} k_{1}}{(2 \pi)^{4}} \frac{\mathrm{d}^{4} k_{2}}{(2 \pi)^{4}} \frac{l \cdot A\left(k_{2}\right) l \cdot A\left(k_{1}\right)}{\left(l \cdot\left(k_{1}+k_{2}\right)+i \epsilon\right)\left(l \cdot k_{1}+i \epsilon\right)} \\
= & e^{2} i^{2} \int_{0}^{\infty} \mathrm{d} \lambda_{2} \int_{\lambda_{2}}^{\infty} \mathrm{d} \lambda_{1} l \cdot A\left(l \lambda_{2}\right) l \cdot \mathcal{A}\left(l \lambda_{1}\right) \\
& =\frac{e^{2} i^{2}}{2 !} \int_{0}^{\infty} \mathrm{d} \lambda_{2} \int_{0}^{\infty} \mathrm{d} \lambda_{1} l \cdot \mathcal{A}\left(l \lambda_{2}\right) l \cdot \mathcal{A}\left(l \lambda_{1}\right) .
\end{aligned}
$$

Extending this calculation up to infinite number of soft photons we find that the eikonal contribution for the infrared divergences due to soft photons exchange with the light-like electron in QED is given by the exponential

$e^{i e \int_{0}^{\infty} \mathrm{d} \lambda l \cdot A(l \lambda)}$

where $l^{\mu}$ is the light-like four-velocity of the electron. The Wilson line in QED is given by

$e^{i e \int_{x_{i}}^{x_{f}} \mathrm{~d} x^{\mu} A_{\mu}(x)}$.

When $A^{\mu}(x)=A^{\mu}(l \lambda)$ as in Eq. (24) then one finds from Eq. (25) that the light-like Wilson line in QED for infrared divergences is given by [48]

$e^{i e \int_{0}^{x} \mathrm{~d} x^{\mu} A_{\mu}(x)}=e^{-i e \int_{0}^{\infty} \mathrm{d} \lambda l \cdot A(x+l \lambda)} e^{i e \int_{0}^{\infty} \mathrm{d} \lambda l \cdot A(l \lambda)}$.

Note that a light-like electron traveling with light-like fourvelocity $l^{\mu}$ produces $\mathrm{U}(1)$ pure gauge potential $A^{\mu}(x)$ at all the time-space position $x^{\mu}$ except at the position $\vec{x}$ perpendicular to the direction of motion of the electron $(\vec{l} \cdot \vec{x}=0)$ at the time of closest approach $[40,49,50]$. When $A^{\mu}(x)=A^{\mu}(\lambda l)$ as in Eq. (24) we find $\vec{l} \cdot \vec{x}=\lambda \vec{l} \cdot \vec{l}=\lambda \neq 0$, which implies that the light-like Wilson line finds the photon field $A^{\mu}(x)$ in Eq. (24) as the U(1) pure gauge. The U(1) pure gauge is given by

$A^{\mu}(x)=\partial^{\mu} \omega(x)$, 
which gives from Eq. (26) the light-like Wilson line in QED for infrared divergences

$$
\begin{aligned}
e^{i e \omega(x)} e^{-i e \omega(0)} & =e^{i e \int_{0}^{x} \mathrm{~d} x^{\mu} A_{\mu}(x)} \\
& =e^{-i e \int_{0}^{\infty} \mathrm{d} \lambda l \cdot A(x+l \lambda)} e^{i e \int_{0}^{\infty} \mathrm{d} \lambda l \cdot A(l \lambda)},
\end{aligned}
$$

which depends only on the end points 0 and $x^{\mu}$ but is independent of the path. The path independence can also be found from Stokes' theorem because for pure gauge

$F^{\mu \nu}(x)=\partial^{\mu} A^{v}(x)-\partial^{v} A^{\mu}(x)=0$,

which gives from Stokes' theorem

$e^{i e \oint_{C} \mathrm{~d} x^{\mu} A_{\mu}(x)}=e^{i e \int_{S} \mathrm{~d} y^{\mu} \mathrm{d} x^{\nu} F_{\mu \nu}(x)}=1$

where $C$ is a closed path and $S$ is the surface enclosing $C$. Now considering two different paths $L$ and $M$ with the common end points 0 and $x^{\mu}$ we find

$e^{i e \oint_{C} \mathrm{~d} x^{\mu} A_{\mu}(x)}=e^{i e \int_{L} \mathrm{~d} x^{\mu} A_{\mu}(x)-i e \int_{M} \mathrm{~d} x^{\mu} A_{\mu}(x)}=1$,

which implies that

$e^{i e \int_{0}^{x} \mathrm{~d} x^{\mu} A_{\mu}(x)}$

depends only on the end points 0 and $x^{\mu}$ but is independent of the path, which can also be seen from Eq. (28). Hence from Eq. (28) we find that the abelian phase or the gauge link in QED is given by

$e^{-i e \int_{0}^{\infty} \mathrm{d} \lambda l \cdot A(x+l \lambda)}=e^{i e \omega(x)}$.

From Eqs. (17) and (33) one expects that the gauge invariance and factorization of infrared divergences in QED can be explained simultaneously.

One can recall that the gauge invariant greens function in QED

$$
\begin{aligned}
G\left(x_{1}, x_{2}\right)= & \langle 0| \bar{\psi}\left(x_{2}\right) \times \exp \left[i e \int_{x_{1}}^{x_{2}} \mathrm{~d} x^{\mu} A_{\mu}(x)\right] \\
& \times \psi\left(x_{1}\right)|0\rangle
\end{aligned}
$$

in the presence of a background field $A^{\mu}(x)$ was formulated by Schwinger long time ago [51,52]. When this background field $A^{\mu}(x)$ is replaced by the $\mathrm{U}(1)$ pure gauge background field as given by Eq. (27) then one finds by using the path integral method of QED that [33]

$$
\begin{aligned}
e^{i e \omega\left(x_{2}\right)} & \left\langle 0\left|\bar{\psi}\left(x_{2}\right) \psi\left(x_{1}\right)\right| 0\right\rangle_{A} e^{-i e \omega\left(x_{1}\right)} \\
= & \left\langle 0\left|\bar{\psi}\left(x_{2}\right) \psi\left(x_{1}\right)\right| 0\right\rangle \\
= & e^{-i e \int_{0}^{\infty} \mathrm{d} \lambda l \cdot A\left(x_{2}+l \lambda\right)}\left\langle 0\left|\bar{\psi}\left(x_{2}\right) \psi\left(x_{1}\right)\right| 0\right\rangle_{A} \\
& \quad \times e^{i e \int_{0}^{\infty} \mathrm{d} \lambda l \cdot A\left(x_{1}+l \lambda\right)},
\end{aligned}
$$

which proves the gauge invariance and factorization of infrared divergences in QED simultaneously. In Eq. (35) the $\left\langle 0\left|\bar{\psi}\left(x_{2}\right) \psi\left(x_{1}\right)\right| 0\right\rangle$ is the full Green's function in QED and $\left\langle 0\left|\bar{\psi}\left(x_{2}\right) \psi\left(x_{1}\right)\right| 0\right\rangle_{A}$ is the corresponding Green's function in the background field method of QED. This path integral technique is also used in [44] to prove factorization of infrared divergences in non-equilibrium QED.

Hence we find that the gauge invariance and factorization of infrared divergences in QED can be studied by using the path integral method of QED in the presence of a U(1) pure gauge background field. Therefore one expects that the gauge invariance and factorization of infrared divergences in QCD can be studied by using the path integral method of QCD in the presence of a SU(3) pure gauge background field.

Now let us proceed to QCD. In QCD the infrared (or soft) divergence arises only from the emission of a gluon for which all components of the four-momentum are small. The eikonal propagator times the eikonal vertex for a soft gluon with momentum $k$ interacting with a light-like quark moving with four-momentum $p^{\mu}$ is given by $[33,34,38-44,47]$

$g T^{a} \frac{p^{\mu}}{p \cdot k+i \epsilon}=g T^{a} \frac{l^{\mu}}{l \cdot k+i \epsilon}$

where $l^{\mu}$ is the four-velocity of the light-like quark. Note that when we talk about the "light-like quark" we mean the quark that is traveling at its highest speed, which is arbitrarily close to the speed of light $(|\vec{l}| \sim 1)$ as it cannot travel exactly at the speed of light $(|\vec{l}|=1)$ because it has a finite mass even if the mass of the light quark is very small. On the other hand the gluon is massless and hence it always travels at the speed of light and is exactly light-like. From Eq. (36) we find

$$
\begin{aligned}
& g T^{a} \int \frac{\mathrm{d}^{4} k}{(2 \pi)^{4}} \frac{l \cdot A^{a}(k)}{l \cdot k+i \epsilon}=-g T^{a} i \int_{0}^{\infty} \mathrm{d} \lambda \\
& \quad \times \int \frac{\mathrm{d}^{4} k}{(2 \pi)^{4}} e^{i l \cdot k \lambda} l \cdot A^{a}(k)=i g T^{a} \int_{0}^{\infty} \mathrm{d} \lambda l \cdot A^{a}(l \lambda)
\end{aligned}
$$

where the gluon field $A^{\mu a}(x)$ and its Fourier transform $A^{\mu a}(k)$ are related by

$A^{\mu a}(x)=\int \frac{\mathrm{d}^{4} k}{(2 \pi)^{4}} A^{\mu a}(k) e^{i k \cdot x}$.

Note that a path ordering in QCD is required, which can be seen as follows; see also [46]. The eikonal contribution for the infrared divergence in QCD arising from a single softgluon exchange in Feynman diagram is given by Eq. (37). Now consider the corresponding Feynman diagram for the infrared divergences in QCD due to the exchange of two soft gluons of four-momenta $k_{1}^{\mu}$ and $k_{2}^{\mu}$. The corresponding eikonal contribution due to the exchange of two soft gluons is analogously given by

$$
\begin{aligned}
& g^{2} \int \frac{\mathrm{d}^{4} k_{1}}{(2 \pi)^{4}} \frac{\mathrm{d}^{4} k_{2}}{(2 \pi)^{4}} \frac{T^{a} l \cdot A^{a}\left(k_{2}\right) T^{b} l \cdot A^{b}\left(k_{1}\right)}{\left(l \cdot\left(k_{1}+k_{2}\right)+i \epsilon\right)\left(l \cdot k_{1}+i \epsilon\right)} \\
& \quad=g^{2} i^{2} \int_{0}^{\infty} \mathrm{d} \lambda_{2} \int_{\lambda_{2}}^{\infty} \mathrm{d} \lambda_{1} T^{a} l \cdot A^{a}\left(l \lambda_{2}\right) T^{b} l \cdot A^{b}\left(l \lambda_{1}\right)
\end{aligned}
$$




$$
=\frac{g^{2} i^{2}}{2 !} \mathcal{P} \int_{0}^{\infty} \mathrm{d} \lambda_{2} \int_{0}^{\infty} \mathrm{d} \lambda_{1} T^{a} l \cdot A^{a}\left(l \lambda_{2}\right) T^{b} l \cdot A^{b}\left(l \lambda_{1}\right)
$$

where $\mathcal{P}$ is the path ordering. Extending this calculation up to an infinite number of soft gluons we find that the eikonal contribution for the infrared divergences due to soft gluons exchange with the light-like quark in QCD is given by the path ordered exponential

$\mathcal{P} \exp \left[i g \int_{0}^{\infty} \mathrm{d} \lambda l \cdot A^{a}(l \lambda) T^{a}\right]$

where $l^{\mu}$ is the light-like four-velocity of the quark. The Wilson line in QCD is given by

$\mathcal{P} e^{i g \int_{x_{i}}^{x} f} \mathrm{~d} x^{\mu} A_{\mu}^{a}(x) T^{a}$,

which is the solution of the equation [53]

$\partial_{\mu} S(x)=i g T^{a} A_{\mu}^{a}(x) S(x)$

with initial condition

$S\left(x_{i}\right)=1$.

When $A^{\mu a}(x)=A^{\mu a}(l \lambda)$ as in Eq. (40) we find from Eq. (41) that the light-like Wilson line in QCD for infrared divergences is given by [48]

$$
\begin{aligned}
& \mathcal{P} e^{i g \int_{0}^{x} \mathrm{~d} x^{\mu} A_{\mu}^{a}(x) T^{a}}=\left[\mathcal{P} e^{-i g \int_{0}^{\infty} \mathrm{d} \lambda l \cdot A^{a}(x+l \lambda) T^{a}}\right] \\
& \times \mathcal{P} e^{i g \int_{0}^{\infty} \mathrm{d} \lambda l \cdot A^{b}(l \lambda) T^{b}} .
\end{aligned}
$$

A light-like quark traveling with light-like four-velocity $l^{\mu}$ produces a SU(3) pure gauge potential $A^{\mu a}(x)$ at all the time-space position $x^{\mu}$ except at the position $\vec{x}$ perpendicular to the direction of motion of the quark $(\vec{l} \cdot \vec{x}=0)$ at the time of closest approach $[40,49,50]$. When $A^{\mu a}(x)=A^{\mu a}(\lambda l)$ as in Eq. (40) we find $\vec{l} \cdot \vec{x}=\lambda \vec{l} \cdot \vec{l}=\lambda \neq 0$, which implies that the light-like Wilson line finds the gluon field $A^{\mu a}(x)$ in Eq. (40) as the $\mathrm{SU}(3)$ pure gauge. The $\mathrm{SU}(3)$ pure gauge is given by

$T^{a} A_{\mu}^{a}(x)=\frac{1}{i g}\left[\partial_{\mu} U(x)\right] U^{-1}(x), \quad U(x)=e^{i g T^{a} \omega^{a}(x)}$,

which gives

$U\left(x_{f}\right)=\mathcal{P} e^{i g \int_{x_{i}}^{x_{f}} \mathrm{~d} x^{\mu} A_{\mu}^{a}(x) T^{a}} U\left(x_{i}\right)=e^{i g T^{a} \omega^{a}\left(x_{f}\right)}$.

Hence when $A^{\mu a}(x)=A^{\mu a}(\lambda l)$ as in Eq. (40) we find from Eqs. (44) and (46) that the light-like Wilson line in QCD for infrared divergences is given by

$$
\begin{aligned}
\mathcal{P} e^{i g \int_{0}^{x} \mathrm{~d} x^{\mu} A_{\mu}^{a}(x) T^{a}}=e^{i g T^{a} \omega^{a}(x)} e^{-i g T^{b} \omega^{b}(0)} \\
=\left[\mathcal{P} e^{-i g \int_{0}^{\infty} \mathrm{d} \lambda l \cdot A^{a}(x+l \lambda) T^{a}}\right] \mathcal{P} e^{i g \int_{0}^{\infty} \mathrm{d} \lambda l \cdot A^{b}(l \lambda) T^{b}},
\end{aligned}
$$

which depends only on the end points 0 and $x^{\mu}$ but is independent of the path. The path independence can also be found from the non-abelian Stokes theorem, which can be seen as follows. The SU(3) pure gauge in Eq. (45) gives

$$
F_{\mu \nu}^{a}[A]=\partial_{\mu} A_{\nu}^{a}(x)-\partial_{\nu} A_{\mu}^{a}(x)+g f^{a b c} A_{\mu}^{b}(x) A_{\nu}^{c}(x)=0 .
$$

Note that from Eq. (48) we find the vanishing physical gauge invariant field strength square $F^{\mu \nu a}[A] F_{\mu \nu}^{a}[A]$ when $A^{\mu a}(x)$ is the $\mathrm{SU}(3)$ pure gauge as given by Eq. (45). Hence in classical mechanics the SU(3) pure gauge potential does not have an effect on color charged particle and one expects the effect of exchange of soft gluons to simply vanish. However, in quantum mechanics the situation is a little more complicated, because the gauge potential does have an effect on color charged particle even if it is a SU(3) pure gauge potential and hence one should not expect the effect of exchange of soft gluons to simply vanish [40]. This can be verified by studying the non-perturbative matrix element in QCD such as $\left\langle\bar{\Psi}(x) \Psi\left(x^{\prime}\right) \bar{\Psi}\left(x^{\prime \prime}\right) \Psi\left(x^{\prime \prime \prime}\right) \cdots\right\rangle$ in the presence of a SU(3) pure gauge background field.

Using Eq. (48) in the non-abelian Stokes theorem [54] we find

$$
\begin{aligned}
& \mathcal{P} e^{i g \oint_{C} \mathrm{~d} x^{\mu} A_{\mu}^{a}(x) T^{a}}=\mathcal{P} \exp \left[i g \int_{S} \mathrm{~d} x^{\mu} \mathrm{d} x^{\nu}\right. \\
& \times\left[\mathcal{P} e^{i g \int_{y}^{x} \mathrm{~d} x^{\prime \lambda} A_{\lambda}^{b}\left(x^{\prime}\right) T^{b}}\right] \\
& \left.\quad \times F_{\mu \nu}^{a}(x) T^{a}\left[\mathcal{P} e^{i g \int_{x}^{y} \mathrm{~d} x^{\prime \prime \delta} A_{\delta}^{c}\left(x^{\prime \prime}\right) T^{c}}\right]\right]=1
\end{aligned}
$$

where $C$ is a closed path and $S$ is the surface enclosing $C$. Now considering two different paths $L$ and $M$ with the common end points 0 and $x^{\mu}$ we find from Eq. (49)

$$
\begin{aligned}
& \mathcal{P} e^{i g \oint_{C} \mathrm{~d} x^{\mu} A_{\mu}^{a}(x) T^{a}} \\
& =\mathcal{P} \exp \left[i g \int_{L} \mathrm{~d} x^{\mu} A_{\mu}^{a}(x) T^{a}-i g \int_{M} \mathrm{~d} x^{\mu} A_{\mu}^{a}(x) T^{a}\right] \\
& \quad=\left[\mathcal{P} e^{i g \int_{L} \mathrm{~d} x^{\mu} A_{\mu}^{a}(x) T^{a}}\right]\left[\mathcal{P} e^{-i g \int_{M} \mathrm{~d} x^{v} A_{\nu}^{b}(x) T^{b}}\right]=1,
\end{aligned}
$$

which implies that the light-like Wilson line in QCD

$\mathcal{P} e^{i g \int_{0}^{x} \mathrm{~d} x^{\mu} A_{\mu}^{a}(x) T^{a}}$

depends only on the end points 0 and $x^{\mu}$ but is independent of the path, which can also be seen from Eq. (47). Hence from Eq. (47) we find that the non-abelian phase or the gauge link in QCD is given by

$$
\Phi(x)=\mathcal{P} e^{-i g \int_{0}^{\infty} \mathrm{d} \lambda l \cdot A^{a}(x+l \lambda) T^{a}}=e^{i g T^{a} \omega^{a}(x)} .
$$

In the adjoint representation of SU(3) the corresponding path ordered exponential is given by 


$$
\begin{gathered}
\mathcal{P} \exp \left[-i g \int_{0}^{\infty} \mathrm{d} \lambda l \cdot A^{c}(x+l \lambda) T^{(A) c}\right]=e^{i g T^{(A) c} \omega^{c}(x)}, \\
\left(T^{(A) c}\right)_{a b}=-i f^{a b c} .
\end{gathered}
$$

To summarize this, we find that the infrared divergences in the perturbative Feynman diagrams due to soft-gluon interaction with the light-like Wilson line in QCD is given by the path ordered exponential in Eq. (40), which is nothing but the non-abelian phase or the gauge link in QCD as given by Eq. (52) where the gluon field $A^{\mu a}(x)$ is the SU(3) pure gauge; see Eqs. (45), (46), (47). This implies that the effect of soft-gluons interaction between the partons and the light-like Wilson line in QCD can be studied by putting the partons in the SU(3) pure gauge background field. Hence we find that the infrared behavior of a non-perturbative matrix element such as $\left\langle 0\left|\bar{\Psi}(x) \Psi\left(x^{\prime}\right) \bar{\Psi}\left(x^{\prime \prime}\right) \Psi\left(x^{\prime \prime \prime}\right) \cdots\right| 0\right\rangle$ in QCD due to the presence of a light-like Wilson line in QCD can be studied by using the path integral method of QCD in the presence of a $\mathrm{SU}(3)$ pure gauge background field.

It can be mentioned here that in soft collinear effective theory (SCET) [55-57] it is also necessary to use the idea of background fields [37] to give a well-defined meaning to several distinct gluon fields [41].

As mentioned earlier, in NRQCD an ultraviolet cutoff $\Lambda \sim M$ is introduced [1]. Hence the ultraviolet (UV) behavior of QCD and NRQCD differ. However, the infrared (IR) behavior of QCD and NRQCD remains same [36]. Hence the infrared behavior in NRQCD can be studied by studying the corresponding infrared behavior in QCD. Hence we find that the infrared behavior of the non-perturbative NRQCD matrix element $\left\langle 0\left|\chi^{\dagger} K_{n} \xi\left(a_{H}^{\dagger} a_{H}\right) \xi^{\dagger} K_{n}^{\prime} \chi\right| 0\right\rangle$ in Eq. (1) can be obtained by studying the infrared behavior of the non-perturbative matrix element in QCD of the type $\left\langle 0\left|\bar{\Psi}(x) O_{n} \Psi(x) \bar{\Psi}\left(x^{\prime}\right) O_{n}^{\prime} \Psi\left(x^{\prime}\right)\right| 0\right\rangle$ where $O_{n}, O_{n}^{\prime}$ are appropriate factors which identify the state of the heavy quarkantiquark system such as the color singlet state or color octet state etc.

Note that a massive color source traveling at a speed much less than the speed of light cannot produce a SU(3) pure gauge field $[40,49,50]$. Hence when one replaces a light-like Wilson line with a massive Wilson line one expects the factorization of infrared divergences to break down. This is in confirmation with the finding in $[58,59]$ which used the diagrammatic method of QCD. In the case of a massive Wilson line in QCD the color transfer occurs and the factorization breaks down.

\section{Eikonal current of the light-like charge generates pure gauge field in quantum field theory}

In order to study factorization of infrared divergences by using the background field method of QED, the soft-photon cloud traversed by the electron is represented by the pure gauge background field $A^{\mu}(x)$ [33] due to the presence of the light-like Wilson line, where one represents the quantum photon field by $Q^{\mu}(x)$. As mentioned above, in classical mechanics we have the assertion that the gauge field that is produced by a highly relativistic (light-like) particle is a pure gauge $[40,49,50]$. One may ask if this assertion is correct in quantum field theory. In this section we will show that this assertion is correct in quantum field theory. We will use the path integral formulation of quantum field theory for this purpose.

The generating functional for the gauge field in quantum field theory in the presence of an external source $J^{\mu}(x)$ in the path integral formulation is given by

$Z[J]=\int[\mathrm{d} Q] e^{i \int \mathrm{d}^{4} x\left[-\frac{1}{4} F_{\mu \nu}^{2}[Q]-\frac{1}{2 \alpha}\left(\partial_{\mu} Q^{\mu}\right)^{2}+J \cdot Q\right]}$

where $Q^{\mu}(x)$ is the quantum photon field and

$$
\begin{gathered}
F^{\mu \nu}[Q]=\partial^{\mu} Q^{\nu}(x)-\partial^{\nu} Q^{\mu}(x), \\
F_{\mu \nu}^{2}[Q]=F^{\mu \nu}[Q] F_{\mu \nu}[Q] .
\end{gathered}
$$

The effective action $S_{\text {eff }}[J]$ is given by $[60,61]$

$$
\langle 0 \mid 0\rangle_{J}=\frac{Z[J]}{Z[0]}=e^{i S_{\mathrm{eff}}[J]}
$$

where

$S_{\text {eff }}[J]=-\frac{1}{2} \int \mathrm{d}^{4} x \mathrm{~d}^{4} x^{\prime} J^{\mu}(x) D_{\mu v}\left(x-x^{\prime}\right) J^{v}\left(x^{\prime}\right)$

where $D_{\mu \nu}\left(x-x^{\prime}\right)$ is the photon propagator.

The photon propagator in the coordinate space is given by

$D_{\mu \nu}\left(x-x^{\prime}\right)=\frac{1}{\partial^{2}}\left[g_{\mu \nu}+\frac{(\alpha-1)}{\partial^{2}} \partial_{\mu} \partial_{\nu}\right] \delta^{(4)}\left(x-x^{\prime}\right)$.

Using Eq. (58) in (57) we find

$S_{\text {eff }}[J]=-\frac{1}{2} \int \mathrm{d}^{4} x J^{\mu}(x) \frac{1}{\partial^{2}}\left[g_{\mu \nu}+\frac{(\alpha-1)}{\partial^{2}} \partial_{\mu} \partial_{\nu}\right] J^{\nu}(x)$.

From the continuity equation we have

$\partial_{\mu} J^{\mu}(x)=0$.

Using Eq. (60) in (59) we find

$S_{\text {eff }}[J]=-\frac{1}{2} \int \mathrm{d}^{4} x J^{\mu}(x) \frac{1}{\partial^{2}} J_{\mu}(x)$.

6.1 Derivation of Coulomb's law for static charge in quantum field theory

First of all, by using the path integral formulation of the quantum field theory we will derive Coulomb's law for static charge. Note that the derivation of the Coulomb law by using the path integral formulation of quantum field theory is not 
necessary to prove factorization theorem. We have included it here only to demonstrate the correctness of the prediction of the path integral formulation in quantum field theory which we will use (see below) to show that the eikonal current of the light-like charge generates pure gauge field in quantum field theory.

In order to derive Coulomb's law by using the path integral formulation of quantum field theory we consider two static charges at positions $\vec{X}$ and $\vec{X}^{\prime}$, respectively. The current density for this two static charges is given by

$J^{\mu}(x)=e \delta^{\mu 0} \delta^{(3)}(\vec{x}-\vec{X})+e \delta^{\mu 0} \delta^{(3)}\left(\vec{x}-\vec{X}^{\prime}\right)$.

Using Eq. (62) in (61) and neglecting the self energies we find in the time interval $t$ that

$$
\begin{aligned}
S_{\mathrm{eff}}^{\mathrm{int}}[J] & =e^{2} \int \mathrm{d} t \frac{1}{\nabla_{X}^{2}} \delta^{(3)}\left(\vec{X}-\vec{X}^{\prime}\right) \\
& =-t e^{2} \frac{1}{\nabla_{X}^{2}} \nabla_{X}^{2}\left(\frac{1}{\left|\vec{X}-\vec{X}^{\prime}\right|}\right)=-t V_{\mathrm{eff}}^{\mathrm{int}}[J],
\end{aligned}
$$

which gives the (effective) potential energy $V_{\text {eff }}[J]$ of the interaction between two static charges to be

$V_{\mathrm{eff}}^{\mathrm{int}}[J]=\frac{e^{2}}{\left|\vec{X}-\vec{X}^{\prime}\right|}$,

which reproduces the Coulomb law. Hence we have shown that the assertion that a charge at rest generates a Coulomb gauge field is correct in quantum field theory.

\subsection{Effective lagrangian density of light-like eikonal current in quantum field theory}

Similarly using the above procedure in quantum field theory we will show that the assertion that a light-like charge generates pure gauge field is correct in quantum field theory. This can be shown as follows.

The eikonal current density of the charge $e$ with lightlike four-velocity $l^{\mu}$ is given by Eq. (22). By using the path integral formulation of quantum field theory we find by using Eq. (22) in (61) that for light-like eikonal current the effective lagrangian density is given by

$\mathcal{L}_{\text {eff }}(x)=\frac{e^{2}}{2} \frac{\left(l^{2}\right)^{2}}{(l \cdot x)^{4}}$.

For light-like four-velocity we have

$l^{2}=l^{\mu} l_{\mu}=0$.

Hence from (65) and (66) we find that for light-like eikonal current the effective lagrangian density is given by

$\mathcal{L}_{\text {eff }}(x)=0, \quad l \cdot x \neq 0$

at all the time-space positions $x^{\mu}$ except at the spatial position perpendicular to the motion of the charge $(\vec{l} \cdot \vec{x}=0)$ at the time of closest approach $\left(x_{0}=0\right)$.
6.3 Interaction between non-eikonal current and the gauge field generated by light-like eikonal current in quantum field theory

Similarly by using the above path integral formulation calculation we find from Eq. (A5) that the interaction between the (light-like or non-light-like) non-eikonal current and the gauge field generated by the light-like eikonal current gives the effective (interaction) lagrangian density

$\mathcal{L}_{\mathrm{eff}}^{\mathrm{int}}(x)=l^{2} \frac{e^{2}}{2} \frac{(l \cdot q)(q \cdot x)-(l \cdot x) q^{2}}{(l \cdot x)^{3}\left[(q \cdot x)^{2}-q^{2} x^{2}\right]^{\frac{3}{2}}}$

where $q^{\mu}$ is the (light-like or non-light-like) four-momentum of non-eikonal current of charge $e$ and $l^{\mu}$ is the light-like four-velocity of the eikonal current of charge $e$.

For light-like eikonal current we find from Eqs. (66) and (68) that effective (interaction) lagrangian density due to the interaction between the (light-like or non-light-like) noneikonal current of four-momentum $q^{\mu}$ and the gauge field generated by the light-like eikonal current of four-velocity $l^{\mu}$ is given by

$\mathcal{L}_{\mathrm{eff}}^{\text {int }}(x)=0$, for $q \cdot x \neq 0, \quad l \cdot x \neq 0$.

This is also obvious from Eq. (81).

6.4 Pure gauge field generated by eikonal current of light-like charge in quantum field theory

Hence from Eqs. (67) and (69) we find that the eikonal current for light-like charge generates pure gauge field in quantum field theory. From Eqs. (67) and (69) we find that the assertion that a light-like charge generates a pure gauge field is correct in quantum field theory which is consistent with the corresponding result in classical mechanics $[40,49,50]$.

\section{Pure gauge field in quantum field theory describes soft (infrared) divergence}

In this section we will show how the pure gauge field is used in quantum field theory to describe soft (infrared) divergences. Consider an incoming electron of four-momentum $q^{\mu}$ and mass $m$ emitting a real photon of four-momentum $k^{\mu}$. The corresponding Feynman diagram contribution is given by [62]

$$
\begin{aligned}
\mathcal{M} & =\frac{1}{\gamma_{\nu} q^{\nu}-\gamma_{\nu} k^{\nu}-m} \gamma_{\mu} \epsilon^{\mu}(k) u(q) \\
& =-\frac{q \cdot \epsilon(k)}{q \cdot k} u(q)+\frac{k^{\nu} \gamma_{\nu} \gamma_{\mu} \epsilon^{\mu}(k)}{2 q \cdot k} u(q)
\end{aligned}
$$

where we write

$\mathcal{M}_{\text {eikonal }}=-\frac{q \cdot \epsilon(k)}{q \cdot k} u(q)$ 
and

$\mathcal{M}_{\text {non-eikonal }}=\frac{k^{\nu} \gamma_{\nu} \gamma_{\mu} \epsilon^{\mu}(k)}{2 q \cdot k} u(q)$.

From Eq. (3.2) of [62] we write the gauge field as

$$
\begin{aligned}
\epsilon^{\mu}(k) & =\left[\epsilon^{\mu}(k)-k^{\mu} \frac{q \cdot \epsilon(k)}{q \cdot k}\right]+k^{\mu} \frac{q \cdot \epsilon(k)}{q \cdot k} \\
& =\epsilon_{\text {phys }}^{\mu}(k)+\epsilon_{\text {pure }}^{\mu}(k)
\end{aligned}
$$

where

$\epsilon_{\text {phys }}^{\mu}(k)=\left[\epsilon^{\mu}(k)-k^{\mu} \frac{q \cdot \epsilon(k)}{q \cdot k}\right]$

is the physical gauge field [corresponding to transverse polarization of the gauge field] and

$\epsilon_{\text {pure }}^{\mu}(k)=k^{\mu} \frac{q \cdot \epsilon(k)}{q \cdot k}$

is the pure gauge field [corresponding to longitudinal polarization of the gauge field].

Now using Eq. (73) in Eq. (70) we find that the total contribution of the Feynman diagram is given by

$\mathcal{M}=\mathcal{M}_{\text {eikonal }}+\mathcal{M}_{\text {non-eikonal }}$

where

$$
\begin{aligned}
\mathcal{M}_{\text {eikonal }} & =-\frac{q \cdot \epsilon_{\mathrm{phys}}(k)}{q \cdot k} u(q)-\frac{q \cdot \epsilon_{\mathrm{pure}}(k)}{q \cdot k} u(q) \\
& =-\frac{q \cdot \epsilon_{\mathrm{pure}}(k)}{q \cdot k} u(q)
\end{aligned}
$$

and

$$
\begin{aligned}
\mathcal{M}_{\text {non-eikonal }} & =\frac{k^{\nu} \gamma_{\nu} \gamma_{\mu} \epsilon_{\mathrm{phys}}^{\mu}(k)}{2 q \cdot k} u(q)+\frac{k^{\nu} \gamma_{\nu} \gamma_{\mu} \epsilon_{\mathrm{pure}}^{\mu}(k)}{2 q \cdot k} u(q) \\
& =\frac{k^{\nu} \gamma_{\nu} \gamma_{\mu} \epsilon_{\mathrm{phys}}^{\mu}(k)}{2 q \cdot k} u(q) .
\end{aligned}
$$

Hence in the soft-photon limit $\left(k_{0}, k_{1}, k_{2}, k_{3}\right) \rightarrow 0$ we find from the Eqs. (70) and (77) that

$$
\begin{gathered}
-\mathcal{M}_{\text {eikonal }}=\frac{q \cdot \epsilon(k)}{q \cdot k} u(q)=\frac{q \cdot \epsilon_{\text {pure }}(k)}{q \cdot k} u(q) \\
\rightarrow \infty \text { as }\left(k_{0}, k_{1}, k_{2}, k_{3}\right) \rightarrow 0,
\end{gathered}
$$

which implies that the physical gauge field [corresponding to transverse polarization] does not contribute to the soft (infrared) divergences in quantum field theory and the soft (infrared) divergences can be calculated by using pure gauge field [corresponding to longitudinal polarization] in quantum field theory.

Similarly from Eqs. (70) and (78) we find that

$$
\begin{gathered}
\mathcal{M}_{\text {non-eikonal }}=\frac{k^{\nu} \gamma_{\nu} \gamma_{\mu} \epsilon^{\mu}(k)}{2 q \cdot k} u(q)=\frac{k^{\nu} \gamma_{\nu} \gamma_{\mu} \epsilon_{\mathrm{phys}}^{\mu}(k)}{2 q \cdot k} u(q) \\
\rightarrow \text { finite as }\left(k_{0}, k_{1}, k_{2}, k_{3}\right) \rightarrow 0,
\end{gathered}
$$

which contributes to the finite part of the cross section which implies that pure gauge field [corresponding to longitudinal polarization] does not contribute to the finite cross section and the finite cross section can be calculated by using physical gauge field [corresponding to transverse polarization].

Hence we find that the non-eikonal-line part of the diagram as given by Eq. (80) is necessary if we are calculating the finite value of the cross section but it is not necessary if we are calculating the relevant infrared divergence behavior. The relevant infrared divergence behavior can be calculated by using the eikonal-line part of the diagram as given by Eq. (79).

For this reason, in the proof of NRQCD factorization of infrared divergences for heavy quarkonium production at NNLO in the coupling constant, the non-eikonal-line part of the diagram as given by Eq. (80) is not considered as the full calculation of the cross section or fragmentation function at NNLO will be daunting but fortunately the analysis of relevant infrared behavior at NNLO requires only the eikonal approximation as given by Eq. (79); see the discussion in the last paragraph of section 4 of [29]. Similarly the full calculation of the cross section or fragmentation function at all orders in the coupling constant by using the non-eikonal-line part of the diagram as given by Eq. (80) will require nonperturbative QCD which is not solved yet but fortunately the analysis of relevant infrared behavior at all orders in the coupling constant requires only the eikonal approximation as given by Eq. (79).

Hence we find that we do not need to calculate the finite value of the cross section (or the full cross section) [which will require the non-eikonal-line part of the diagram as given by Eq. (80)] to study the relevant infrared divergence behavior. The relevant infrared divergence behavior can be calculated by using eikonal approximation as given by Eq. (79).

From Eq. (80) we find that

$\mathcal{M}_{\text {non-eikonal }}^{\text {pure gauge field }}=\frac{k^{\nu} \gamma_{\nu} \gamma_{\mu} \epsilon_{\text {pure }}^{\mu}(k)}{2 q \cdot k} u(q)=0$.

We are interested in the infrared divergence behavior due to the presence of the light-like Wilson line. We have shown in Eqs. (67) and (69) that the eikonal current of the light-like charge generates pure gauge field in quantum field theory. Hence from Eqs. (67), (79), (69), and (81) we find that the soft (infrared) divergence behavior due to the presence lightlike Wilson line can be studied by using pure gauge field in quantum field theory without modifying the finite value of the cross section. 
8 Heavy quark-antiquark non-perturbative matrix element in the presence of a light-like Wilson line in QCD

We have seen in Sect. 5 that the infrared behavior of the nonperturbative NRQCD matrix element $\langle 0| \chi^{\dagger} K_{n} \xi\left(a_{H}^{\dagger} a_{H}\right) \xi^{\dagger}$ $K_{n}^{\prime} \chi|0\rangle$ in Eq. (1) can be obtained by studying the infrared behavior of the non-perturbative matrix element in QCD of the type $\left\langle 0\left|\bar{\Psi}(x) O_{n} \Psi(x) \bar{\Psi}\left(x^{\prime}\right) O_{n}^{\prime} \Psi\left(x^{\prime}\right)\right| 0\right\rangle$ where $O_{n}, O_{n}^{\prime}$ are appropriate factors which identify the state of the heavy quark-antiquark system such as the color singlet state or the color octet state etc. Similarly, we have also seen in Sect. 5 that the infrared behavior of the non-perturbative matrix element in QCD of the type $\left\langle 0\left|\bar{\Psi}(x) O_{n} \Psi(x) \bar{\Psi}\left(x^{\prime}\right) O_{n}^{\prime} \Psi\left(x^{\prime}\right)\right| 0\right\rangle$ due to the presence of a light-like Wilson line in QCD can be studied by using the path integral method of QCD in the presence of a $\mathrm{SU}(3)$ pure gauge background field. Hence we use the path integral formulation of the background field method of QCD to study non-perturbative matrix element $\left\langle 0\left|\bar{\Psi}(x) O_{n} \Psi(x) \bar{\Psi}\left(x^{\prime}\right) O_{n}^{\prime} \Psi\left(x^{\prime}\right)\right| 0\right\rangle$ in QCD in the presence of a SU(3) pure gauge background field as given by Eq. (45).

The background field method of QCD was originally formulated by 't Hooft [63] and later extended by KluebergStern and Zuber [64,65] and by Abbott [37]. This is an elegant formalism which can be useful to construct gauge invariant non-perturbative green's functions in QCD. This formalism is also useful to study quark and gluon production from the classical chromofield $[60,61]$ via the Schwinger mechanism [66], to compute $\beta$ function in QCD [67], to perform calculations in lattice gauge theories [68] and to study evolution of QCD coupling constant in the presence of a chromofield [69].

In the background field method of QCD the generating functional is given by [37,63-65] the background field by $A^{\mu a}$. The determinant $\operatorname{det}\left(\frac{\delta G^{a}(Q)}{\delta \omega^{b}}\right)$ in Eq. (82) can be expressed in terms of path integration over the ghost fields $[35,64,65]$. However, we will directly work with the determinant $\operatorname{det}\left(\frac{\delta G^{a}(Q)}{\delta \omega^{b}}\right)$ in Eq. (82).

Note that the gauge fixing term $\frac{1}{2 \alpha}\left(G^{a}(Q)\right)^{2}$ in Eq. (82) [where $G^{a}(Q)$ is given by Eq. (83)] is invariant for gauge transformation of $A_{\mu}^{a}$ :

$\delta A_{\mu}^{a}=g f^{a b c} A_{\mu}^{b} \omega^{c}+\partial_{\mu} \omega^{a}, \quad$ (type I transformation),

provided one also performs a homogeneous transformation of $Q_{\mu}^{a}[37,64,65]$ :

$\delta Q_{\mu}^{a}=g f^{a b c} Q_{\mu}^{b} \omega^{c}$.

The gauge transformation of the background field $A_{\mu}^{a}$ as given by Eq. (85) along with the homogeneous transformation of $Q_{\mu}^{a}$ in Eq. (86) gives

$\delta\left(A_{\mu}^{a}+Q_{\mu}^{a}\right)=g f^{a b c}\left(A_{\mu}^{b}+Q_{\mu}^{b}\right) \omega^{c}+\partial_{\mu} \omega^{a}$,

which leaves $-\frac{1}{4} F_{\mu \nu}^{a 2}[A+Q]$ invariant in Eq. (82).

For fixed $A_{\mu}^{a}$, i.e., for

$\delta A_{\mu}^{a}=0, \quad$ (type II transformation)

the gauge transformation of $Q_{\mu}^{a}[37,64,65]$ :

$\delta Q_{\mu}^{a}=g f^{a b c}\left(A_{\mu}^{b}+Q_{\mu}^{b}\right) \omega^{c}+\partial_{\mu} \omega^{a}$

gives Eq. (87), which leaves $-\frac{1}{4} F_{\mu \nu}^{a 2}[A+Q]$ invariant in Eq. (82).

Extending Eq. (82) to include the heavy quark [by using the lagrangian density from Eq. (6)] we find that the generating functional in the background field method of QCD is given by

$Z[A, J, \eta, \bar{\eta}]=\int[\mathrm{d} Q][\mathrm{d} \bar{\psi}][\mathrm{d} \psi] \operatorname{det}\left(\frac{\delta G^{a}(Q)}{\delta \omega^{b}}\right) e^{i \int \mathrm{d}^{4} x\left[-\frac{1}{4} F_{\mu \nu}^{a 2}[A+Q]-\frac{1}{2 \alpha}\left(G^{a}(Q)\right)^{2}+\bar{\psi}\left[i \gamma^{\mu} \partial_{\mu}-m+g T^{a} \gamma^{\mu}(A+Q)_{\mu}^{a}\right] \psi+J \cdot Q+\bar{\eta} \psi+\bar{\psi} \eta\right]}$

where $Q^{\mu a}(x)$ is the quantum gluon field and the gauge fixing term is given by

$G^{a}(Q)=\partial_{\mu} Q^{\mu a}+g f^{a b c} A_{\mu}^{b} Q^{\mu c}=D_{\mu}[A] Q^{\mu a}$,

which depends on the background field $A^{\mu a}$ and

$$
\begin{aligned}
F_{\mu \nu}^{a}[A+Q]= & \partial_{\mu}\left[A_{\nu}^{a}+Q_{\nu}^{a}\right]-\partial_{\nu}\left[A_{\mu}^{a}+Q_{\mu}^{a}\right] \\
& +g f^{a b c}\left[A_{\mu}^{b}+Q_{\mu}^{b}\right]\left[A_{\nu}^{c}+Q_{\nu}^{c}\right] .
\end{aligned}
$$

We have followed the notations of [37,63-65] and accordingly we have denoted the quantum gluon field by $Q^{\mu a}$ and

$$
\begin{aligned}
& Z\left[A, J, \eta_{u}, \bar{\eta}_{u}, \eta_{d}, \bar{\eta}_{d}, \eta_{s}, \bar{\eta}_{s}, \eta_{h}, \bar{\eta}_{h}\right] \\
& =\int[\mathrm{d} Q]\left[\mathrm{d} \bar{\psi}_{1}\right]\left[\mathrm{d} \psi_{1}\right]\left[\mathrm{d} \bar{\psi}_{2}\right]\left[\mathrm{d} \psi_{2}\right]\left[\mathrm{d} \bar{\psi}_{3}\right]\left[\mathrm{d} \psi_{3}\right][\mathrm{d} \bar{\Psi}][\mathrm{d} \Psi] \\
& \quad \times \operatorname{det}\left(\frac{\delta G^{a}(Q)}{\delta \omega^{b}}\right) \\
& \quad \times \exp \left[i \int d ^ { 4 } x \left[-\frac{1}{4} F_{\mu \nu}^{a 2}[A+Q]-\frac{1}{2 \alpha}\left(G^{a}(Q)\right)^{2}\right.\right. \\
& \quad+J \cdot Q+\sum_{l=1}^{3}\left[\bar{\psi}_{l}\left[i \gamma^{\mu} \partial_{\mu}-m_{l}+g T^{a} \gamma^{\mu}(A+Q)_{\mu}^{a}\right] \psi_{l}\right. \\
& \left.\quad+\bar{\eta}_{l} \psi_{l}+\bar{\psi}_{l} \eta_{l}\right]+\bar{\Psi}\left[i \gamma^{\mu} \partial_{\mu}-M\right. \\
& \left.\left.\left.\quad+g T^{a} \gamma^{\mu}(A+Q)_{\mu}^{a}\right] \Psi+\bar{\eta}_{h} \Psi+\bar{\Psi} \eta_{h}\right]\right] .
\end{aligned}
$$


Note that in the absence of external sources a pure gauge can be gauged away from the generating functional. However, in the presence of external sources a pure gauge cannot be gauged away from the generating functional. It is useful to remember that, unlike QED [33], finding an exact relation between the generating functional $Z\left[J, \eta_{u}, \bar{\eta}_{u}, \eta_{d}, \bar{\eta}_{d}, \eta_{s}, \bar{\eta}_{s}, \eta_{h}, \eta_{h}\right]$ in QCD in Eq. (12) and the generating functional $Z\left[A, J, \eta_{u}, \bar{\eta}_{u}, \eta_{d}, \bar{\eta}_{d}, \eta_{s}, \bar{\eta}_{s}, \eta_{h}\right.$, $\eta_{h}$ ] in the background field method of QCD in Eq. (90) in the presence of a SU(3) pure gauge background field is not easy. The main difficulty is due to the gauge fixing terms, which are different in the two cases. While the Lorentz (covariant) gauge fixing term $-\frac{1}{2 \alpha}\left(\partial_{\mu} Q^{\mu a}\right)^{2}$ in Eq. (12) in QCD is independent of the background field $A^{\mu a}(x)$, the background field gauge fixing term $-\frac{1}{2 \alpha}\left(G^{a}(Q)\right)^{2}$ in Eq. (90) in the background field method of QCD depends on the background field $A^{\mu a}(x)$ where $G^{a}(Q)$ is given by Eq. (83) [37,6365]. Hence in order to study the non-perturbative matrix element in the background field method of QCD in the presence of a $\mathrm{SU}(3)$ pure gauge background field we proceed as follows.

By changing $Q \rightarrow Q-A$ in Eq. (90) we find that
Note that

$A_{\mu}^{\prime a}(x)=A_{\mu}^{a}(x)+g f^{a b c} \omega^{c}(x) A_{\mu}^{b}(x)+\partial_{\mu} \omega^{a}(x)$

in Eq. (85) is valid for an infinitesimal transformation $(\omega<<$ 1 ), which is obtained from the finite equation

$$
\begin{aligned}
T^{a} A_{\mu}^{\prime a}(x) & =U(x) T^{a} A_{\mu}^{a}(x) U^{-1}(x)+\frac{1}{i g}\left[\partial_{\mu} U(x)\right] U^{-1}(x), \\
U(x) & =e^{i g T^{a} \omega^{a}(x)} .
\end{aligned}
$$

Simplifying infinite numbers of non-commuting terms we find

$$
\left[e^{-i g T^{b} \omega^{b}(x)} T^{a} e^{i g T^{c} \omega^{c}(x)}\right]_{i j}=\left[e^{-g M(x)}\right]_{a b} T_{i j}^{b}
$$

where

$M_{a b}(x)=f^{a b c} \omega^{c}(x)$.

Hence from Eqs. (95), (96), and [49] we find that

$$
\begin{aligned}
A_{\mu}^{\prime a}(x)= & {\left[e^{g M(x)}\right]_{a b} A_{\mu}^{b}(x) } \\
& +\left[\frac{e^{g M(x)}-1}{g M(x)}\right]_{a b}\left[\partial_{\mu} \omega^{b}(x)\right]
\end{aligned}
$$

$Z\left[A, J, \eta_{u}, \bar{\eta}_{u}, \eta_{d}, \bar{\eta}_{d}, \eta_{s}, \bar{\eta}_{s}, \eta_{h}, \bar{\eta}_{h}\right]=e^{-i \int \mathrm{d}^{4} x J \cdot A} \int[\mathrm{d} Q]\left[\mathrm{d} \bar{\psi}_{1}\right]\left[\mathrm{d} \psi_{1}\right]\left[\mathrm{d} \bar{\psi}_{2}\right]\left[\mathrm{d} \psi_{2}\right]\left[\mathrm{d} \bar{\psi}_{3}\right]\left[\mathrm{d} \psi_{3}\right][\mathrm{d} \bar{\Psi}][\mathrm{d} \Psi] \operatorname{det}\left(\frac{\delta G_{f}^{a}(Q)}{\delta \omega^{b}}\right)$

$$
\times e^{i \int \mathrm{d}^{4} x\left[-\frac{1}{4} F^{a}{ }_{\mu \nu}^{2}[Q]-\frac{1}{2 \alpha}\left(G_{f}^{a}(Q)\right)^{2}+J \cdot Q+\sum_{l=1}^{3}\left[\bar{\psi}_{l}\left[i \gamma^{\mu} \partial_{\mu}-m_{l}+g T^{a} \gamma^{\mu} Q_{\mu}^{a}\right] \psi_{l}+\bar{\eta}_{l} \psi_{l}+\eta_{l} \bar{\psi}_{l}\right]+\bar{\Psi}\left[i \gamma^{\mu} \partial_{\mu}-M+g T^{a} \gamma^{\mu} Q_{\mu}^{a}\right] \Psi+\bar{\eta}_{h} \Psi+\bar{\Psi} \eta_{h}\right]}
$$

where the gauge fixing term from Eq. (83) becomes

$$
\begin{aligned}
G_{f}^{a}(Q) & =\partial_{\mu} Q^{\mu a}+g f^{a b c} A_{\mu}^{b} Q^{\mu c}-\partial_{\mu} A^{\mu a} \\
& =D_{\mu}[A] Q^{\mu a}-\partial_{\mu} A^{\mu a},
\end{aligned}
$$

and Eq. (86) [by using Eq. (85), a type I transformation [37, $64,65]$ becomes

$\delta Q_{\mu}^{a}=g f^{a b c} Q_{\mu}^{b} \omega^{c}+\partial_{\mu} \omega^{a}$.

Equations (92) and (93) can also be derived by using a type II transformation, which can be seen as follows. By changing $Q \rightarrow Q-A$ in Eq. (90) we find Eq. (91) where the gauge fixing term from Eq. (83) becomes Eq. (92) and Eq. (89) [by using Eq. (88)] becomes Eq. (93). Hence we obtain Eqs. (91), (92), and (93) whether we use the type I transformation or type II transformation. Hence we find that we will obtain the same Eq. (120) whether we use the type I transformation or type II transformation. where $M_{a b}(x)$ is given by Eq. (97). Similarly, the equation

$$
Q_{\mu}^{\prime a}(x)=Q_{\mu}^{a}(x)+g f^{a b c} \omega^{c}(x) Q_{\mu}^{b}(x)+\partial_{\mu} \omega^{a}(x)
$$

in Eq. (93) is valid for an infinitesimal transformation $(\omega<<$ 1 ), which is obtained from the finite equation

$$
\begin{aligned}
T^{a} Q_{\mu}^{\prime a}(x)= & U(x) T^{a} Q_{\mu}^{a}(x) U^{-1}(x) \\
& +\frac{1}{i g}\left[\partial_{\mu} U(x)\right] U^{-1}(x),
\end{aligned}
$$

which gives

$$
\begin{aligned}
Q_{\mu}^{\prime a}(x)= & {\left[e^{g M(x)}\right]_{a b} Q_{\mu}^{b}(x) } \\
& +\left[\frac{e^{g M(x)}-1}{g M(x)}\right]_{a b}\left[\partial_{\mu} \omega^{b}(x)\right]
\end{aligned}
$$

where $M_{a b}(x)$ is given by Eq. (97).

Changing the integration variables from unprimed to primed variables in Eq. (91) we find 
$Z\left[A, J, \eta_{u}, \bar{\eta}_{u}, \eta_{d}, \bar{\eta}_{d}, \eta_{s}, \bar{\eta}_{s}, \eta_{h}, \bar{\eta}_{h}\right]=e^{-i \int \mathrm{d}^{4} x J \cdot A} \int\left[\mathrm{d} Q^{\prime}\right]\left[\mathrm{d} \bar{\psi}_{1}^{\prime}\right]\left[\mathrm{d} \psi_{1}^{\prime}\right]\left[\mathrm{d} \bar{\psi}_{2}^{\prime}\right]\left[\mathrm{d} \psi_{2}^{\prime}\right]\left[\mathrm{d} \bar{\psi}_{3}^{\prime}\right]\left[\mathrm{d} \psi_{3}^{\prime}\right]\left[\mathrm{d} \bar{\Psi}^{\prime}\right]\left[\mathrm{d} \Psi^{\prime}\right] \operatorname{det}\left(\frac{\delta G_{f}^{a}\left(Q^{\prime}\right)}{\delta \omega^{b}}\right)$ $\times e^{i \int \mathrm{d}^{4} x\left[-\frac{1}{4} F^{a}{ }_{\mu \nu}^{2}\left[Q^{\prime}\right]-\frac{1}{2 \alpha}\left(G_{f}^{a}\left(Q^{\prime}\right)\right)^{2}+J \cdot Q^{\prime}+\sum_{l=1}^{3}\left[\bar{\psi}_{l}^{\prime}\left[i \gamma^{\mu} \partial_{\mu}-m_{l}+g T^{a} \gamma^{\mu} Q_{\mu}^{\prime a}\right] \psi_{l}^{\prime}+\bar{\eta}_{l} \psi_{l}^{\prime}+\bar{\psi}_{l}^{\prime} \eta_{l}\right]+\bar{\Psi}^{\prime}\left[i \gamma^{\mu} \partial_{\mu}-M+g T^{a} \gamma^{\mu} Q_{\mu}^{\prime a}\right] \Psi^{\prime}+\bar{\eta}_{h} \Psi^{\prime}+\bar{\Psi}^{\prime} \eta_{h}\right]}$.

This is because a change of variables from unprimed to primed variables does not change the value of the integration.

Under the finite transformation, using Eq. (101), we find

$$
\begin{aligned}
{\left[\mathrm{d} Q^{\prime}\right] } & =[\mathrm{d} Q] \operatorname{det}\left[\frac{\partial Q^{\prime a}}{\partial Q^{b}}\right]=[\mathrm{d} Q] \operatorname{det}\left[e^{g M(x)}\right] \\
& =[\mathrm{d} Q] \exp \left[\operatorname{Tr}\left(\ln \left[e^{g M(x)}\right]\right)\right]=[\mathrm{d} Q]
\end{aligned}
$$

where we have used (for any matrix $H$ )

$\operatorname{det} H=\exp [\operatorname{Tr}(\ln H)]$.

Similarly the fermion fields transform accordingly, see Eq. (16), i.e.,

$\psi_{l}^{\prime}(x)=e^{i g T^{a} \omega^{a}(x)} \psi_{l}(x), \quad \Psi^{\prime}(x)=e^{i g T^{a} \omega^{a}(x)} \Psi(x)$.

Using Eqs. (101) and (105) we find

$\left[\mathrm{d} \bar{\psi}_{1}^{\prime}\right]\left[\mathrm{d} \psi_{1}^{\prime}\right]=\left[\mathrm{d} \bar{\psi}_{1}\right]\left[\mathrm{d} \psi_{1}\right], \quad\left[\mathrm{d} \bar{\psi}_{2}^{\prime}\right]\left[\mathrm{d} \psi_{2}^{\prime}\right]=\left[\mathrm{d} \bar{\psi}_{2}\right]\left[\mathrm{d} \psi_{2}\right]$,

$\left[\mathrm{d} \bar{\psi}_{3}^{\prime}\right]\left[\mathrm{d} \psi_{3}^{\prime}\right]=\left[\mathrm{d} \bar{\psi}_{3}\right]\left[\mathrm{d} \psi_{3}\right],\left[\mathrm{d} \bar{\Psi}^{\prime}\right]\left[\mathrm{d} \Psi^{\prime}\right]=[\mathrm{d} \bar{\Psi}][\mathrm{d} \Psi]$,

$\bar{\psi}_{l}^{\prime}\left[i \gamma^{\mu} \partial_{\mu}-m_{l}+g T^{a} \gamma^{\mu} Q_{\mu}^{\prime a}\right] \psi_{l}^{\prime}$

$=\bar{\psi}_{l}\left[i \gamma^{\mu} \partial_{\mu}-m_{l}+g T^{a} \gamma^{\mu} Q_{\mu}^{a}\right] \psi_{l}$,

$\bar{\Psi}^{\prime}\left[i \gamma^{\mu} \partial_{\mu}-M+g T^{a} \gamma^{\mu} Q_{\mu}^{\prime a}\right] \Psi^{\prime}$

$=\bar{\Psi}\left[i \gamma^{\mu} \partial_{\mu}-m_{l}+g T^{a} \gamma^{\mu} Q_{\mu}^{a}\right] \Psi$,

$F_{\mu \nu}^{a 2}\left[Q^{\prime}\right]=F_{\mu \nu}^{a 2}[Q]$.

Using Eqs. (103) and (106) in Eq. (102) we find
$A^{\mu a}(x)=\partial^{\mu} \omega^{b}(x)\left[\frac{e^{g M(x)}-1}{g M(x)}\right]_{a b}$

where $M_{a b}(x)$ is given by Eq. (97). By using Eqs. (101) and (109) in Eq. (108) we find

$$
\begin{aligned}
G_{f}^{a}\left(Q^{\prime}\right)= & \partial^{\mu}\left[\left[e^{g M(x)}\right]_{a b} Q_{\mu}^{b}(x)\right. \\
& \left.+\left[\frac{e^{g M(x)}-1}{g M(x)}\right]_{a b}\left[\partial_{\mu} \omega^{b}(x)\right]\right] \\
& +g f^{a b c}\left[\partial^{\mu} \omega^{e}(x)\left[\frac{e^{g M(x)}-1}{g M(x)}\right]_{b e}\right] \\
& \times\left[\left[e^{g M(x)}\right]_{c d} Q_{\mu}^{d}(x)\right. \\
& \left.+\left[\frac{e^{g M(x)}-1}{g M(x)}\right]_{c d}\left[\partial_{\mu} \omega^{d}(x)\right]\right] \\
& -\partial_{\mu}\left[\partial^{\mu} \omega^{b}(x)\left[\frac{e^{g M(x)}-1}{g M(x)}\right]_{a b}\right],
\end{aligned}
$$

which gives

$$
\begin{aligned}
& G_{f}^{a}\left(Q^{\prime}\right)=\partial^{\mu}\left[\left[e^{g M(x)}\right]_{a b} Q_{\mu}^{b}(x)\right] \\
& \quad+g f^{a b c}\left[\partial^{\mu} \omega^{e}(x)\left[\frac{e^{g M(x)}-1}{g M(x)}\right]_{b e}\right]\left[\left[e^{g M(x)}\right]_{c d} Q_{\mu}^{d}(x)\right. \\
& \left.\quad+\left[\frac{e^{g M(x)}-1}{g M(x)}\right]_{c d}\left[\partial_{\mu} \omega^{d}(x)\right]\right] .
\end{aligned}
$$

From Eq. (111) we find

$Z\left[A, J, \eta_{u}, \bar{\eta}_{u}, \eta_{d}, \bar{\eta}_{d}, \eta_{s}, \bar{\eta}_{s}, \eta_{h}, \bar{\eta}_{h}\right]=e^{-i \int d^{4} x J \cdot A} \int[d Q]\left[d \bar{\psi}_{1}\right]\left[d \psi_{1}\right]\left[d \bar{\psi}_{2}\right]\left[d \psi_{2}\right]\left[d \bar{\psi}_{3}\right]\left[d \psi_{3}\right][d \bar{\Psi}][d \Psi] \operatorname{det}\left(\frac{\delta G_{f}^{a}\left(Q^{\prime}\right)}{\delta \omega^{b}}\right)$ $e^{i \int d^{4} x\left[-\frac{1}{4} F^{a}{ }_{\mu \nu}^{2}[Q]-\frac{1}{2 \alpha}\left(G_{f}^{a}\left(Q^{\prime}\right)\right)^{2}+J \cdot Q^{\prime}+\sum_{l=1}^{3}\left[\bar{\psi}_{l}\left[i \gamma^{\mu} \partial_{\mu}-m_{l}+g T^{a} \gamma^{\mu} Q_{\mu}^{a}\right] \psi_{l}+\bar{\eta}_{l} \psi_{l}^{\prime}+\bar{\psi}_{l}^{\prime} \eta_{l}\right]+\bar{\Psi}\left[i \gamma^{\mu} \partial_{\mu}-M+g T^{a} \gamma^{\mu} Q_{\mu}^{a}\right] \Psi+\bar{\eta}_{h} \Psi^{\prime}+\bar{\Psi}^{\prime} \eta_{h}\right]}$.

From Eq. (92) we find

$G_{f}^{a}\left(Q^{\prime}\right)=\partial_{\mu} Q^{\prime \mu a}+g f^{a b c} A_{\mu}^{b} Q^{\prime \mu c}-\partial_{\mu} A^{\mu a}$.

By simplifying the infinite number of non-commuting terms in the SU(3) pure gauge in Eq. (45) we find [49]

$$
\begin{gathered}
G_{f}^{a}\left(Q^{\prime}\right)=\partial^{\mu}\left[\left[e^{g M(x)}\right]_{a b} Q_{\mu}^{b}(x)\right]+g f^{a b c}\left[\partial^{\mu} \omega^{e}(x)\right. \\
\left.\times\left[\frac{e^{g M(x)}-1}{g M(x)}\right]_{b e}\right]_{\left[\left[e^{g M(x)}\right]_{c d} Q_{\mu}^{d}(x)\right],}
\end{gathered}
$$


which gives

$$
\begin{aligned}
& G_{f}^{a}\left(Q^{\prime}\right)=\left[e^{g M(x)}\right]_{a b} \partial^{\mu} Q_{\mu}^{b}(x) \\
& \quad+Q_{\mu}^{b}(x) \partial^{\mu}\left[\left[e^{g M(x)}\right]_{a b}\right]+\left[\partial^{\mu} \omega^{e}(x)\left[\frac{e^{g M(x)}-1}{g M(x)}\right]_{b e}\right] \\
& g f^{a b c}\left[\left[e^{g M(x)}\right]_{c d} Q_{\mu}^{d}(x)\right] .
\end{aligned}
$$

From [49] we find

$$
\begin{aligned}
& \partial^{\mu}\left[e^{i g T^{a} \omega^{a}(x)}\right]_{i j}=i g\left[\partial^{\mu} \omega^{b}(x)\right]\left[\frac{e^{g M(x)}-1}{g M(x)}\right]_{a b} \\
& \quad \times T_{i k}^{a}\left[e^{i g T^{c} \omega^{c}(x)}\right]_{k j},
\end{aligned}
$$

we find from Eq. (116) that

$$
\begin{aligned}
\operatorname{det} & {\left[\frac{\delta G_{f}^{a}\left(Q^{\prime}\right)}{\delta \omega^{b}}\right]=\operatorname{det}\left[\frac{\delta\left[\left[e^{g M(x)}\right]_{a c} \partial^{\mu} Q_{\mu}^{c}(x)\right]}{\delta \omega^{b}}\right] } \\
& =\operatorname{det}\left[\left[e^{g M(x)}\right]_{a c} \frac{\delta\left(\partial^{\mu} Q_{\mu}^{c}(x)\right)}{\delta \omega^{b}}\right] \\
& =\left[\operatorname{det}\left[\left[e^{g M(x)}\right]_{a c}\right]\right]\left[\operatorname{det}\left[\frac{\delta\left(\partial^{\mu} Q_{\mu}^{c}(x)\right)}{\delta \omega^{b}}\right]\right] \\
& =\exp \left[\operatorname{Tr}\left(\ln \left[e^{g M(x)}\right]\right)\right] \operatorname{det}\left[\frac{\delta\left(\partial_{\mu} Q^{\mu a}(x)\right)}{\delta \omega^{b}}\right] \\
& =\operatorname{det}\left[\frac{\delta\left(\partial_{\mu} Q^{\mu a}(x)\right)}{\delta \omega^{b}}\right] .
\end{aligned}
$$

Using Eqs. (117) and (119) in Eq. (107) we find

$Z\left[A, J, \eta_{u}, \bar{\eta}_{u}, \eta_{d}, \bar{\eta}_{d}, \eta_{s}, \bar{\eta}_{s}, \eta_{h}, \bar{\eta}_{h}\right]=e^{-i \int \mathrm{d}^{4} x J \cdot A} \int[\mathrm{d} Q]\left[\mathrm{d} \bar{\psi}_{1}\right]\left[\mathrm{d} \psi_{1}\right]\left[\mathrm{d} \bar{\psi}_{2}\right]\left[\mathrm{d} \psi_{2}\right]\left[\mathrm{d} \bar{\psi}_{3}\right]\left[\mathrm{d} \psi_{3}\right][\mathrm{d} \bar{\Psi}][\mathrm{d} \Psi] \operatorname{det}\left[\frac{\delta\left(\partial_{\mu} Q^{\mu a}(x)\right)}{\delta \omega^{b}}\right]$ $\times e^{i \int \mathrm{d}^{4} x\left[-\frac{1}{4} F^{a}{ }_{\mu \nu}^{2}[Q]-\frac{1}{2 \alpha}\left(\partial_{\mu} Q^{\mu a}\right)^{2}+J \cdot Q^{\prime}+\sum_{l=1}^{3}\left[\bar{\psi}_{l}\left[i \gamma^{\mu} \partial_{\mu}-m_{l}+g T^{a} \gamma^{\mu} Q_{\mu}^{a}\right] \psi_{l}+\bar{\eta}_{l} \psi_{l}^{\prime}+\bar{\psi}_{l}^{\prime} \eta_{l}\right]+\bar{\Psi}\left[i \gamma^{\mu} \partial_{\mu}-M+g T^{a} \gamma^{\mu} Q_{\mu}^{a}\right] \Psi+\bar{\eta}_{h} \Psi^{\prime}+\bar{\Psi}^{\prime} \eta_{h}\right] .}$

which in the adjoint representation of SU(3) gives (by using $\left.T_{b c}^{a}=-i f^{a b c}\right)$

$\left[\partial^{\mu} e^{g M(x)}\right]_{a d}=\left[\partial^{\mu} \omega^{e}(x)\right]\left[\frac{e^{g M(x)}-1}{g M(x)}\right]_{b e} g f^{b a c}\left[e^{M(x)}\right]_{c d}$

where $M_{a b}(x)$ is given by Eq. (97). Using Eq. (115) in (113) we find

$G_{f}^{a}\left(Q^{\prime}\right)=\left[e^{g M(x)}\right]_{a b} \partial^{\mu} Q_{\mu}^{b}(x)$,

which gives

$\left(G_{f}^{a}\left(Q^{\prime}\right)\right)^{2}=\left(\partial_{\mu} Q^{\mu a}(x)\right)^{2}$.

Since for $n \times n$ matrices $A$ and $B$ we have

$\operatorname{det}(A B)=(\operatorname{det} A)(\operatorname{det} B)$,
From Eqs. (109) and (101) we find

$Q^{\prime a}(x)-A_{\mu}^{a}(x)=\left[e^{g M(x)}\right]_{a b} Q_{\mu}^{b}(x)$

where $M_{a b}(x)$ is given by Eq. (97).

Note that Eqs. (120), (121), and (16) are valid whether we use type I transformation [see Eqs. (85) and (86)] or type II transformation [see Eqs. (88) and (89)].

However, since Eq. (95) is used to study the gauge transformation of the Wilson line in QCD, we will use type I transformation [see Eqs. (85) and (86)] in the rest of the paper, which for the finite transformation gives $[37,64,65]$

$J_{\mu}^{\prime a}(x)=\left[e^{g M(x)}\right]_{a b} J_{\mu}^{b}(x)$

where $M_{a b}(x)$ is given by Eq. (97). From Eqs. (120), (121), and (122) we find

$Z\left[A, J^{\prime}, \eta_{u}, \bar{\eta}_{u}, \eta_{d}, \bar{\eta}_{d}, \eta_{s}, \bar{\eta}_{s}, \eta_{h}, \bar{\eta}_{h}\right]=\int[\mathrm{d} Q]\left[\mathrm{d} \bar{\psi}_{1}\right]\left[\mathrm{d} \psi_{1}\right]\left[\mathrm{d} \bar{\psi}_{2}\right]\left[\mathrm{d} \psi_{2}\right]\left[\mathrm{d} \bar{\psi}_{3}\right]\left[\mathrm{d} \psi_{3}\right][\mathrm{d} \bar{\Psi}][\mathrm{d} \Psi] \operatorname{det}\left[\frac{\delta\left(\partial_{\mu} Q^{\mu a}(x)\right)}{\delta \omega^{b}}\right]$ $\times e^{i \int \mathrm{d}^{4} x\left[-\frac{1}{4} F_{\mu \nu}^{a 2}[Q]-\frac{1}{2 \alpha}\left(\partial_{\mu} Q^{\mu a}\right)^{2}+J \cdot Q+\sum_{l=1}^{3}\left[\bar{\psi}_{l}\left[i \gamma^{\mu} \partial_{\mu}-m_{l}+g T^{a} \gamma^{\mu} Q_{\mu}^{a}\right] \psi_{l}+\bar{\eta}_{l} \psi_{l}^{\prime}+\bar{\psi}_{l}^{\prime} \eta_{l}\right]+\bar{\Psi}\left[i \gamma^{\mu} \partial_{\mu}-M+g T^{a} \gamma^{\mu} Q_{\mu}^{a}\right] \Psi+\bar{\eta}_{h} \Psi^{\prime}+\bar{\Psi}^{\prime} \eta_{h}\right]}$. 
Under the non-abelian gauge transformation the fermion sources transform as $[37,64,65]$

$\eta_{l}^{\prime}(x)=e^{i g T^{a} \omega^{a}(x)} \eta_{l}(x), \quad \eta_{h}^{\prime}(x)=e^{i g T^{a} \omega^{a}(x)} \eta_{h}(x)$.

From Eqs. (105) and (124) we find

$\bar{\eta}_{l}^{\prime} \psi_{l}^{\prime}=\bar{\eta}_{l} \psi_{l}, \quad \bar{\psi}_{l}^{\prime} \eta_{l}^{\prime}=\bar{\psi}_{l} \eta_{l}, \quad \bar{\eta}_{h}^{\prime} \Psi^{\prime}=\bar{\eta}_{h} \Psi$

$$
\bar{\Psi}^{\prime} \eta_{h}^{\prime}=\bar{\Psi} \eta_{h},
$$

which gives from Eq. (123) in the background field method of QCD is given by [33]

$$
\begin{aligned}
& \left\langle 0\left|\bar{\Psi}(x) O_{n} \Psi(x) \bar{\Psi}\left(x^{\prime}\right) O_{n}^{\prime} \Psi\left(x^{\prime}\right)\right| 0\right\rangle_{A} \\
& =\frac{\delta}{\delta \eta_{h}(x)} O_{n} \frac{\delta}{\delta \bar{\eta}_{h}(x)} \frac{\delta}{\delta \eta_{h}\left(x^{\prime}\right)} O_{n}^{\prime} \frac{\delta}{\delta \bar{\eta}_{h}\left(x^{\prime}\right)} \\
& \quad \times Z\left[A, J, \eta_{u}, \bar{\eta}_{u}, \eta_{d}, \bar{\eta}_{d}, \eta_{s},\right. \\
& \left.\quad \bar{\eta}_{s}, \eta_{h}, \bar{\eta}_{h}\right]\left.\right|_{J=\eta_{u}=\bar{\eta}_{u}=\eta_{d}=\bar{\eta}_{d}=\eta_{s}=\bar{\eta}_{s}=\eta_{h}=\eta_{h}=0}
\end{aligned}
$$

where the suppression of the normalization factor $Z[0]$ is understood as it will cancel in the final result (see Eq. (133)).

When the background field $A^{\mu a}(x)$ is the $\mathrm{SU}(3)$ pure gauge as given by Eq. (45) we find from Eqs. (15), (129), (127), (124), and (122) that

$$
\begin{aligned}
& Z\left[A, J^{\prime}, \eta_{u}^{\prime}, \bar{\eta}_{u}^{\prime}, \eta_{d}^{\prime}, \bar{\eta}_{d}^{\prime}, \eta_{s}^{\prime}, \bar{\eta}_{s}^{\prime}, \eta_{h}^{\prime}, \bar{\eta}_{h}^{\prime}\right]=\int[\mathrm{d} Q]\left[\mathrm{d} \bar{\psi}_{1}\right]\left[\mathrm{d} \psi_{1}\right]\left[\mathrm{d} \bar{\psi}_{2}\right]\left[\mathrm{d} \psi_{2}\right]\left[\mathrm{d} \bar{\psi}_{3}\right]\left[\mathrm{d} \psi_{3}\right][\mathrm{d} \bar{\Psi}][\mathrm{d} \Psi] \operatorname{det}\left[\frac{\delta\left(\partial_{\mu} Q^{\mu a}(x)\right)}{\delta \omega^{b}}\right] \\
& \quad \times e^{i \int d^{4} x\left[-\frac{1}{4} F_{\mu \nu}^{a 2}{ }_{\mu \nu}[Q]-\frac{1}{2 \alpha}\left(\partial_{\mu} Q^{\mu a}\right)^{2}+J \cdot Q+\sum_{l=1}^{3}\left[\bar{\psi}_{l}\left[i \gamma^{\mu} \partial_{\mu}-m_{l}+g T^{a} \gamma^{\mu} Q_{\mu}^{a}\right] \psi_{l}+\bar{\eta}_{l} \psi_{l}+\bar{\psi}_{l} \eta_{l}\right]+\bar{\Psi}\left[i \gamma^{\mu} \partial_{\mu}-M+g T^{a} \gamma^{\mu} Q_{\mu}^{a}\right] \Psi+\bar{\eta}_{h} \Psi+\bar{\Psi} \eta_{h}\right] .} .
\end{aligned}
$$

Hence from Eqs. (126) and (12) we find

$$
\begin{aligned}
& Z\left[J, \eta_{u}, \bar{\eta}_{u}, \eta_{d}, \bar{\eta}_{d}, \eta_{s}, \bar{\eta}_{s}, \eta_{h}, \bar{\eta}_{h}\right] \\
& \quad=Z\left[A, J^{\prime}, \eta_{u}^{\prime}, \bar{\eta}_{u}^{\prime}, \eta_{d}^{\prime}, \bar{\eta}_{d}^{\prime}, \eta_{s}^{\prime}, \bar{\eta}_{s}^{\prime}, \eta_{h}^{\prime}, \bar{\eta}_{h}^{\prime}\right]
\end{aligned}
$$

when the background field $A^{\mu a}(x)$ is the $\mathrm{SU}(3)$ pure gauge field as given by Eq. (45).

Hence we find that Eq. (127) is the relation between the generating functional $Z\left[J, \eta_{u}, \bar{\eta}_{u}, \eta_{d}, \bar{\eta}_{d}, \eta_{s}, \bar{\eta}_{s}, \eta_{h}, \bar{\eta}_{h}\right]$ in QCD and the generating functional $Z\left[A, J, \eta_{u}, \bar{\eta}_{u}, \eta_{d}, \bar{\eta}_{d}\right.$, $\left.\eta_{s}, \bar{\eta}_{s}, \eta_{h}, \bar{\eta}_{h}\right]$ in the background field method of QCD in the presence of a SU(3) pure gauge background field $A^{\mu a}(x)$ as given by Eq. (45).

Note that in QED the corresponding result is $[33,44]$

$$
Z[J, \eta, \bar{\eta}]=Z\left[A, J, \eta^{\prime}, \bar{\eta}^{\prime}\right]
$$

when the background field $A^{\mu}(x)$ is the $\mathrm{U}(1)$ pure gauge field given by $A^{\mu}(x)=\partial^{\mu} \omega(x)$. Equation (35) in QED is obtained from Eq. (128). Note that unlike Eq. (127) in QCD there is no $J^{\prime}$ in Eq. (128) in QED because, while the (quantum) gluon directly interacts with the classical chromo-electromagnetic field, the (quantum) photon does not directly interact with the classical electromagnetic field.

Equation (127) is the main result of this paper.

For the heavy quark Dirac field $\Psi(x)$, the non-perturbative matrix element of the type $\langle 0| \bar{\Psi}(x) O_{n} \Psi(x) \bar{\Psi}\left(x^{\prime}\right) O_{n}^{\prime}$ $\Psi\left(x^{\prime}\right)|0\rangle$ in QCD is given by Eq. (15) if the factors $O_{n}$ and $O_{n}^{\prime}$ are independent of quantum fields. Similarly for the heavy quark Dirac field $\Psi(x)$, the corresponding non-perturbative matrix element of the type $\left\langle 0\left|\bar{\Psi}(x) O_{n} \Psi(x) \bar{\Psi}\left(x^{\prime}\right) O_{n}^{\prime} \Psi\left(x^{\prime}\right)\right| 0\right\rangle$

$$
\begin{aligned}
& \left\langle 0\left|\bar{\Psi}(x) O_{n} \Psi(x) \bar{\Psi}\left(x^{\prime}\right) O_{n}^{\prime} \Psi\left(x^{\prime}\right)\right| 0\right\rangle \\
& =\langle 0| \bar{\Psi}(x) \Phi(x) O_{n} \Phi^{\dagger}(x) \Psi(x) \bar{\Psi}\left(x^{\prime}\right) \Phi\left(x^{\prime}\right) \\
& \quad \times O_{n}^{\prime} \Phi^{\dagger}\left(x^{\prime}\right) \Psi\left(x^{\prime}\right)|0\rangle_{A}
\end{aligned}
$$

if the factors $O_{n}$ and $O_{n}^{\prime}$ are independent of quantum fields where, see Eq. (52),

$\Phi(x)=\exp \left[i g T^{a} \omega^{a}(x)\right]=\mathcal{P} e^{-i g \int_{0}^{\infty} \mathrm{d} \lambda l \cdot A^{a}(x+l \lambda) T^{a}}$.

Note that the creation operator $a_{q}^{\dagger}$ and the annihilation operator $a_{q}$ of the quark are related to the quark field via the equation [70]

$$
\begin{gathered}
\psi(x)=\sum_{\text {spin }} \sum_{p} \sqrt{\frac{m}{V E_{p}}}\left[a_{q}(p) u(p) e^{-i p \cdot x}\right. \\
\left.+a_{\bar{q}}^{\dagger}(p) v(p) e^{i p \cdot x}\right]
\end{gathered}
$$

where color indices are suppressed. Hence one finds that the quark field $\psi(x)$ or $\Psi(x)$ depends on the creation (annihilation) operator $a_{q}^{\dagger}\left(a_{q}\right)$ of the quark but is independent of the creation (annihilation) operator $a_{H}^{\dagger}\left(a_{H}\right)$ of the hadron. Similarly the gluon field $Q^{\mu a}(x)$ is independent of the creation (annihilation) operator $a_{H}^{\dagger}\left(a_{H}\right)$ of the hadron. Since $a_{H}^{\dagger} a_{H}$ is independent of $\psi(x), \Psi(x), Q^{\mu a}(x)$ one can perform exactly similar steps to the path integral calculation as above to find from Eq. (130) that

$$
\begin{aligned}
\left\langle 0\left|\bar{\Psi}(x) O_{n} \Psi(x) a_{H}^{\dagger} a_{H} \bar{\Psi}\left(x^{\prime}\right) O_{n}^{\prime} \Psi\left(x^{\prime}\right)\right| 0\right\rangle & \\
= & \langle 0| \bar{\Psi}(x) \Phi(x) O_{n} \Phi^{\dagger}(x) \Psi(x) a_{H}^{\dagger} a_{H} \bar{\Psi}\left(x^{\prime}\right) \\
& \times \Phi\left(x^{\prime}\right) O_{n}^{\prime} \Phi^{\dagger}\left(x^{\prime}\right) \Psi\left(x^{\prime}\right)|0\rangle_{A}
\end{aligned}
$$

where $\Phi(x)$ is given by Eq. (131). 
Under non-abelian gauge transformation as given by Eq. (95) the Wilson line in QCD transforms as

$$
\begin{aligned}
& \mathcal{P} e^{i g \int_{x_{i}}^{x_{f}} \mathrm{~d} x^{\mu} A_{\mu}^{\prime a}(x) T^{a}} \\
&=U\left(x_{f}\right)\left[\mathcal{P} e^{i g \int_{x_{i}}^{x_{f}} \mathrm{~d} x^{\mu} A_{\mu}^{a}(x) T^{a}}\right] U^{-1}\left(x_{i}\right) .
\end{aligned}
$$

From Eqs. (47) and (134) we find

$\mathcal{P} e^{-i g \int_{0}^{\infty} \mathrm{d} \lambda l \cdot A^{\prime a}(x+l \lambda) T^{a}}=U(x) \mathcal{P} e^{-i g \int_{0}^{\infty} \mathrm{d} \lambda l \cdot A^{a}(x+l \lambda) T^{a}}$,

$U(x)=\exp \left[\operatorname{ig} T^{a} \omega^{a}(x)\right]$,

which gives from Eq. (131)

$\Phi^{\prime}(x)=U(x) \Phi(x), \quad \Phi^{\prime \dagger}(x)=\Phi^{\dagger}(x) U^{-1}(x)$.

Hence we find that $\langle 0| \bar{\Psi}(x) \Phi(x) O_{n} \Phi^{\dagger}(x) \Psi(x) a_{H}^{\dagger} a_{H} \bar{\Psi}\left(x^{\prime}\right)$ $\Phi\left(x^{\prime}\right) O_{n}^{\prime} \Phi^{\dagger}\left(x^{\prime}\right) \Psi\left(x^{\prime}\right)|0\rangle_{A}$ in Eq. (133) is gauge invariant and Eq. (133) is consistent with the factorization of infrared divergences in QCD.

\section{Proof of the factorization in heavy quarkonium production in the NRQCD color octet mechanism at all orders in the coupling constant}

The non-perturbative matrix element $\langle 0| \bar{\Psi}(x) O_{n} \Psi(x) a_{H}^{\dagger}$ $a_{H} \bar{\Psi}\left(x^{\prime}\right) O_{n}^{\prime} \Psi\left(x^{\prime}\right)|0\rangle$ in QCD in Eq. (133) is obtained from the exact generating functional in QCD as given by Eq. (12); see Eq. (15). Similarly, the non-perturbative matrix element $\left\langle 0\left|\bar{\Psi}(x) O_{n} \Psi(x) a_{H}^{\dagger} a_{H} \bar{\Psi}\left(x^{\prime}\right) O_{n}^{\prime} \Psi\left(x^{\prime}\right)\right| 0\right\rangle_{A}$ in the background field method of QCD in Eq. (133) is obtained from the exact generating functional in the background field method of QCD as given by Eq. (90); see Eq. (129). Hence we find that Eq. (133) is valid at all orders in the coupling constant in QCD.

Note that in $[29,30]$ the proof of the factorization is presented at NNLO in the coupling constant and to $v^{2}$ order in the relative velocity of the heavy quark-antiquark pair. This is done by restricting the result to $v^{2}$ order by using

$p_{1}^{\mu}=\frac{P^{\mu}}{2}+p_{r}^{\mu}, \quad p_{2}^{\mu}=\frac{P^{\mu}}{2}-p_{r}^{\mu}$

where $p_{1}^{\mu}$ is the momentum of the heavy quark, $p_{2}^{\mu}$ is the momentum of the heavy antiquark, $P^{\mu}$ is the total momentum of the heavy quark-antiquark pair and $p_{r}^{\mu}$ is the relative momentum of the heavy quark-antiquark pair. In the rest frame of the heavy quark-antiquark pair $\vec{p}_{r}=M \vec{v}$ where $M$ is the mass of the heavy quark.

Similarly in [31] the proof of the factorization is presented at NNLO in the coupling constant and to all powers in the relative velocity $v$ of the heavy quark-antiquark pair. This is done by obtaining the result for arbitrary $p_{1}^{\mu}$ and $p_{2}^{\mu}$ without restricting to order $p_{r}^{2}$. Hence in order to be consistent with the proof of the factorization of [31] to all powers of the relative velocity $v$ it is necessary to present the final result for arbitrary $p_{1}^{\mu}$ and $p_{2}^{\mu}$.

It can be seen that the non-perturbative matrix element $\left\langle 0\left|\bar{\Psi}(x) O_{n} \Psi(x) a_{H}^{\dagger} a_{H} \bar{\Psi}\left(x^{\prime}\right) O_{n}^{\prime} \Psi\left(x^{\prime}\right)\right| 0\right\rangle$ in QCD in Eq. (133) is obtained from the exact generating functional in QCD as given by Eq. (12) without putting any restrictions on the heavy quark and antiquark momenta; see Eq. (15). Similarly, the non-perturbative matrix element $\left\langle 0\left|\bar{\Psi}(x) O_{n} \Psi(x) a_{H}^{\dagger} a_{H} \bar{\Psi}\left(x^{\prime}\right) O_{n}^{\prime} \Psi\left(x^{\prime}\right)\right| 0\right\rangle_{A}$ in the background field method of QCD in Eq. (133) is obtained from the exact generating functional in the background field method of QCD as given by Eq. (90) without putting any restrictions on the heavy quark and antiquark momenta; see Eq. (129). Hence we find that Eq. (133) is valid for any arbitrary momenta $p_{1}^{\mu}$ and $p_{2}^{\mu}$ of the heavy quark and antiquark, respectively. This implies that Eq. (133) is valid to all powers in the relative velocity of the heavy quark.

Hence we find that Eq. (133) is valid at all orders in the coupling constant in QCD and to all powers in the relative velocity of the heavy quark.

As mentioned earlier, in NRQCD an ultraviolet cutoff $\Lambda \sim M$ is introduced [1]. Hence the ultraviolet (UV) behavior of QCD and NRQCD differ. However, the infrared (IR) behavior of QCD and NRQCD remains same [36]. Hence the infrared behavior of the non-perturbative NRQCD matrix element $\left\langle 0\left|\chi^{\dagger} K_{n} \xi\left(a_{H}^{\dagger} a_{H}\right) \xi^{\dagger} K_{n}^{\prime} \chi\right| 0\right\rangle$ in Eq. (1) can be obtained by studying the infrared behavior of the non-perturbative matrix element in QCD of the type $\left\langle 0\left|\bar{\Psi}(x) O_{n} \Psi(x) a_{H}^{\dagger} a_{H} \bar{\Psi}\left(x^{\prime}\right) O_{n}^{\prime} \Psi\left(x^{\prime}\right)\right| 0\right\rangle$ where $O_{n}, O_{n}^{\prime}$ are appropriate factors which identify the state of the heavy quark-antiquark system such as the color singlet state or the color octet state etc.

We are interested in the effect of exchange of soft gluons between the light-like Wilson line and the heavy quark (and/or antiquark) in the NRQCD color octet mechanism [29-31]. Hence for the color singlet S-wave non-perturbative matrix element we find from Eq. (133) that

$$
\left\langle 0\left|\mathcal{O}_{n}\right| 0\right\rangle=\left\langle 0\left|\chi^{\dagger}(0) K_{n} \xi(0)\left(a_{H}^{\dagger} a_{H}\right) \xi^{\dagger}(0) K_{n}^{\prime} \chi(0)\right| 0\right\rangle
$$

at all orders in the coupling constant which is consistent with Eq. (1).

When the factors $O_{n}, O_{n}^{\prime}$ contain the color matrix $T^{a}$ we find by simplifying infinite numbers of non-commuting terms [see Eq. (96)] that Eqs. (133) and (131) give

$$
\begin{aligned}
& \left\langle 0\left|\bar{\Psi}(x) O_{n, a} \Psi(x) a_{H}^{\dagger} a_{H} \bar{\Psi}\left(x^{\prime}\right) O_{n, a}^{\prime} \Psi\left(x^{\prime}\right)\right| 0\right\rangle \\
& =\langle 0| \bar{\Psi}(x) O_{n, e} \Psi(x) \Phi_{e b}^{(A) \dagger}(x) a_{H}^{\dagger} a_{H} \Phi^{(A)}\left(x^{\prime}\right)_{b a} \\
& \quad \bar{\Psi}\left(x^{\prime}\right) O_{n, a}^{\prime} \Psi\left(x^{\prime}\right)|0\rangle_{A}
\end{aligned}
$$

where

$$
\begin{aligned}
& \Phi^{(A)}(x)=\mathcal{P} e^{-i g \int_{0}^{\infty} \mathrm{d} \lambda l \cdot A^{a}(x+l \lambda) T^{(A) a}}, \\
& \left(T^{(A) c}\right)_{a b}=-i f^{a b c} .
\end{aligned}
$$


Hence from Eqs. (139) and (140) we find that the gauge invariant octet $\mathrm{S}$-wave non-perturbative NRQCD matrix element which is consistent with factorization of infrared divergences at all orders in the coupling constant and to all powers in the relative velocity of the heavy quark is given by

$$
\begin{gathered}
\left\langle 0\left|\mathcal{O}_{n}\right| 0\right\rangle=\langle 0| \chi^{\dagger}(0) K_{n, e} \xi(0) \Phi_{l}^{(A) \dagger}(0)_{e b}\left(a_{H}^{\dagger} a_{H}\right) \\
\Phi_{l}^{(A)}(0)_{b a} \xi^{\dagger}(0) K_{n, a}^{\prime} \chi(0)|0\rangle
\end{gathered}
$$

where

$$
\begin{aligned}
& \Phi_{l}^{(A)}(0)=\mathcal{P} \exp \left[-i g T^{(A) c} \int_{0}^{\infty} \mathrm{d} \lambda l \cdot A^{c}(l \lambda)\right] \\
& \left(T^{(A) c}\right)_{a b}=-i f^{a b c} .
\end{aligned}
$$

Note that the non-perturbative matrix element $\langle 0| \bar{\Psi}(x) O_{n} \Psi$ $(x) a_{H}^{\dagger} a_{H} \bar{\Psi}\left(x^{\prime}\right) O_{n}^{\prime} \Psi\left(x^{\prime}\right)|0\rangle$ in the left hand side of Eq. (133) is independent of $l^{\mu}$. Hence all the $l^{\mu}$ dependence in $\Phi(x)$ defined by Eq. (131) in the non-perturbative matrix element $\langle 0| \bar{\Psi}(x) \Phi(x) O_{n} \Phi^{\dagger}(x) \Psi(x) a_{H}^{\dagger} a_{H} \bar{\Psi}\left(x^{\prime}\right) \Phi\left(x^{\prime}\right) O_{n}^{\prime} \Phi^{\dagger}\left(x^{\prime}\right) \Psi$ $\left(x^{\prime}\right)|0\rangle_{A}$ in the right hand side of Eq. (133) is canceled by the use of background field $A^{\mu a}(x)$ in the expectation value of the non-perturbative matrix element $\langle 0| \bar{\Psi}(x) O_{n} \Psi(x) a_{H}^{\dagger} a_{H} \bar{\Psi}$ $\left(x^{\prime}\right) O_{n}^{\prime} \Psi\left(x^{\prime}\right)|0\rangle_{A}$ as defined in Eq. (129) in the background field method of QCD. This proves that the long-distance behavior of the non-perturbative NRQCD matrix element $\langle 0| \chi^{\dagger}(0) K_{n, e} \xi(0) \Phi_{l}^{(A) \dagger}(0)_{e b}\left(a_{H}^{\dagger} a_{H}\right) \Phi_{l}^{(A)}(0)_{b a} \xi^{\dagger}(0) K_{n, a}^{\prime} \chi$ (0) $|0\rangle$ in Eq. (141) is independent of the light-like vector $l^{\mu}$ at all orders in the coupling constant and to all powers in the relative velocity of the heavy quark.

To summarize this, we find that Eq. (141), which is found by using the path integral method of QCD, is valid at all orders in the coupling constant and to all powers in the relative velocity of the heavy quark. We have also shown that the long-distance behavior of the non-perturbative NRQCD matrix element is independent of the light-like vector $l^{\mu}$ at all orders in the coupling constant and to all powers in the relative velocity of the heavy quark. Equation (4), which is found by using diagrammatic method of QCD at NNLO in the coupling constant and to all powers in the relative velocity of the heavy quark shows that long-distance behavior of the non-perturbative NRQCD matrix element is independent of the light-like vector $l^{\mu}$ at NNLO in the coupling constant and to all powers in the relative velocity of the heavy quark. This implies that the gauge invariance and the factorization at all orders in the coupling constant require gauge-completed octet $\mathrm{S}$-wave non-perturbative NRQCD matrix element that was introduced previously to prove factorization at NNLO.

Hence we find that Eq. (4) is valid at all orders in the coupling constant and to all powers in the relative velocity of the heavy quark.

\section{Factorization theorem is a key ingredient in calculation of NRQCD heavy quarkonium production cross section}

As mentioned earlier the definition of the NRQCD heavy quarkonium production matrix element from heavy quarkantiquark pair is a non-perturbative quantity, which cannot be calculated by using perturbation theory no matter how many orders of perturbation theory is used. From this point of view the path integral formulation (as opposed to diagrammatic methods in perturbation theory) is useful to study the properties of the NRQCD non-perturbative matrix element of heavy quarkonium production at all orders in the coupling constant. As mentioned earlier the only path integral formulation to study factorization of soft and collinear divergences at all orders in the coupling constant in quantum field theory available is by Tucci [33]. However, the calculation of Tucci [33] was exact for QED but was not exact for QCD. We have extended the exact path integral calculation of the proof of the factorization of Tucci in QED [33] to the proof of the factorization in QCD at all orders in the coupling constant in [34] and to the proof of the factorization in NRQCD heavy quarkonium production at all orders in the coupling constant in the previous section.

In this section we will show how the factorization theorem as given by Eqs. (130) and (139) are actually a key ingredients in calculation of NRQCD heavy quarkonium production by using Eqs. (2) and (3) where the NRQCD non-perturbative matrix element of heavy quarkonium production in the color octet mechanism is given by Eq. (141).

Let us prove how the Eqs. (130) and (139) are key ingredients to prove Eqs. (2) and (3) to calculate the NRQCD heavy quarkonium production in the color octet mechanism. Suppose we calculate the cross section of heavy quark-antiquark production in the color octet state in the presence of a lightlike quark (or gluon). Then from Eq. (130) we find

$$
\begin{aligned}
& \left\langle 0\left|\bar{\Psi}(x) O_{n} \Psi(x) \bar{\Psi}\left(x^{\prime}\right) O_{n}^{\prime} \Psi\left(x^{\prime}\right)\right| 0\right\rangle_{A} \\
& =\langle 0| \bar{\Psi}(x) \Phi^{\dagger}(x) O_{n} \Phi(x) \Psi(x) \bar{\Psi}\left(x^{\prime}\right) \Phi^{\dagger}\left(x^{\prime}\right) \\
& \quad \times O_{n}^{\prime} \Phi\left(x^{\prime}\right) \Psi\left(x^{\prime}\right)|0\rangle .
\end{aligned}
$$

Hence from Eq. (143) we find that the $A$ dependence which arises due to the soft gluon exchanges with light-like quark (or gluon) is factorized and only appears in the gauge-links $\Phi(x)$ in the right hand side where $\Phi(x)$ is given by Eq. (131). Equation (143) implies that in the cross section for $Q \bar{Q}$ production in the color octet state at all orders in the coupling constant the infrared divergences due to the presence of a light-like quark (or gluon) are factorized only to the gauge links $\Phi(x)$.

Equation (143) is the exact extension of eq. (1.6) of [33] of the factorization in QED. 
Hence from Eq. (143) we find that the non-perturbative matrix element of NRQCD heavy quarkonium production in the color octet mechanism which cancels these infrared divergences and is consistent with the factorization theorem is obtained from Eq. (139) and is given by Eq. (141). This proves that the factorization theorem as given by Eqs. (130) and (139) is actually a key ingredient to prove Eqs. (2) and (3) to calculate the NRQCD heavy quarkonium production cross section in the color octet mechanism at all orders in the coupling constant.

\section{Conclusions}

Recently the proof of the factorization in heavy quarkonium production in the NRQCD color octet mechanism is given at next-to-next-to-leading order (NNLO) in the coupling constant by using diagrammatic method of QCD. In this paper we have proved factorization in heavy quarkonium production in the NRQCD color octet mechanism at all orders in the coupling constant by using the path integral method of QCD. Our proof is valid to all powers in the relative velocity of the heavy quark. We have found that the gauge invariance and the factorization at all orders in the coupling constant require gauge-completed non-perturbative NRQCD matrix elements that were introduced previously to prove factorization at NNLO.

Acknowledgments I thank George Sterman for useful discussions and suggestions.

Open Access This article is distributed under the terms of the Creative Commons Attribution 4.0 International License (http://creativecomm ons.org/licenses/by/4.0/), which permits unrestricted use, distribution, and reproduction in any medium, provided you give appropriate credit to the original author(s) and the source, provide a link to the Creative Commons license, and indicate if changes were made.

Funded by SCOAP ${ }^{3}$.

\section{Appendix A: Interaction between non-eikonal current and the gauge field generated by light-like eikonal cur- rent in quantum field theory}

From the non-eikonal part of the diagram in Eq. (72) we find

$e \int \frac{\mathrm{d}^{4} k}{(2 \pi)^{4}} \frac{k^{\nu} \gamma_{\nu} \gamma_{\mu} A^{\mu}(k)}{2 q \cdot k+i \epsilon}=\int \mathrm{d}^{4} x J_{\mu}(x) A^{\mu}(x)$

where the non-eikonal current density $J^{\mu}(x)$ of the (light-like or non-light-like) charge $e$ of four-momentum $q^{\mu}$ is given by

$J^{\mu}(x)=\frac{e}{2} \gamma_{\nu} \gamma^{\mu} \int_{0}^{\infty} \mathrm{d} \lambda \frac{\partial}{\partial x_{\nu}} \delta^{(4)}(x-q \lambda)$.

Hence using Eqs. (A2) and (22) in Eq. (61) we find that the interaction between the non-eikonal current and the gauge field generated by the light-like eikonal current in quantum field theory gives the effective (interaction) action

$$
\begin{aligned}
S_{\text {eff }}^{\text {int }} & J]=l_{\mu} \frac{e^{2}}{4} \int \mathrm{d}^{4} x \gamma_{\nu} \gamma^{\mu} \int_{0}^{\infty} \mathrm{d} \lambda \delta^{(4)}(x-q \lambda) \\
& \times \frac{\partial}{\partial x_{\nu}} \frac{1}{\partial^{2}} \int \mathrm{d} \lambda^{\prime} \delta^{(4)}\left(x-l \lambda^{\prime}\right) \\
= & l^{2} \frac{e^{2}}{2} \int \mathrm{d}^{4} x\left[\frac{l \cdot \partial\left[q \cdot\left(x-q \lambda_{0}\right)\right]}{\left[q \cdot\left(x-q \lambda_{0}\right)\right]^{2}}\right]\left[\frac{1}{(l \cdot x)^{3}}\right]
\end{aligned}
$$

where $\lambda_{0}$ is the solution of the equation

$$
\left(x-q \lambda_{0}\right)^{\mu}\left(x-q \lambda_{0}\right)_{\mu}=0 .
$$

From Eqs. (A3) and (A4) we find that the interaction between the non-light-like non-eikonal current and the gauge field generated by the light-like eikonal current in quantum field theory gives the effective (interaction) lagrangian density

$\mathcal{L}_{\text {eff }}^{\text {int }}(x)=l^{2} \frac{e^{2}}{2} \frac{(l \cdot q)(q \cdot x)-(l \cdot x) q^{2}}{(l \cdot x)^{3}\left[(q \cdot x)^{2}-q^{2} x^{2}\right]^{\frac{3}{2}}}$.

From Eq. (A5) we find that the interaction between the lightlike non-eikonal current and the gauge field generated by the light-like eikonal current in quantum field theory gives the effective (interaction) lagrangian density

$\mathcal{L}_{\mathrm{eff}}^{\mathrm{int}}(x)=\frac{e^{2}}{2} \frac{l^{2}(q \cdot l)}{(q \cdot x)^{2}(l \cdot x)^{3}}$.

\section{References}

1. G.T. Bodwin, E. Braaten, G.P. Lepage, Phys. Rev. D 51, 1125 (1995). Erratum ibid. D55 (1997) 5853. arXiv:hep-ph/9407339

2. F. Abe et al. [CDF Collaboration], Phys. Rev. Lett. 79, 572 (1997)

3. F. Abe et al. [CDF Collaboration], Phys. Rev. Lett. 79, 578 (1997)

4. F. Abe et al. [CDF Collaboration], Phys. Rev. Lett. 75, 4358 (1995)

5. B. Abbott et al. [D0 Collaboration], Phys. Rev. Lett. 82, 35 (1999)

6. T. Affolder et al. [CDF Collaboration], Phys. Rev. Lett. 85, 2886 (2000)

7. T. Affolder et al. [CDF Collaboration], Phys. Rev. Lett. 86, 3963 (2001)

8. D. Acosta et al. [CDF Collaboration], Phys. Rev. Lett. 88, 161802 (2002)

9. D. Acosta et al. [CDF Collaboration], Phys. Rev. D 66, 092001 (2002)

10. D. Acosta et al. [CDF Collaboration], Phys. Rev. D 71, 032001 (2005)

11. ATLAS Collaboration, arXiv:1407.5532 [hep-ex]

12. CMS Collaboration, Phys. Lett. B 727, 101 (2013)

13. LHCb Collaboration, Eur. Phys. C 74, 2835 (2014)

14. ALICE Collaboration, Eur. Phys. C 74, 2974 (2014)

15. B. Fulsom, arXiv:1409.2601 [hep-ex]

16. LHCb Collaboration, Eur. Phys. C 73, 2631 (2013)

17. CMS Collaboration, JHEP 02, 011 (2012)

18. LHCb Collaboration, Eur. Phys. C 72, 2100 (2012)

19. ATLAS Collaboration, Phys. Rev. D 87, 052004 (2014)

20. ATLAS Collaboration, arXiv:1404.7035 [hep-ex]

21. CMS Collaboration, Eur. Phys. C 72, 2251 (2012)

22. LHCb Collaboration, JHEP 10, 115 (2013)

23. CMS Collaboration, CMS-PAS-BPH-13-005 
24. F. Adad et al. [ATLAS Collaboration], ATLAS Note ATLASCONF-2010-062

25. J. Kirk [ATLAS Collaboration], PoS(ICHEP 2010) 013

26. V. Khachatryan et al. [CMS Collaboration], Eur. Phys. C 71, 1575 (2011)

27. E. Scomparin [ALICE Collaboration], Nucl. Phys. B (Proc. Suppl.) 214, 56 (2011)

28. R. Aajj et al. [LHCb Collaboration], Eur. Phys. C 71, 1645 (2011)

29. G.C. Nayak, J. Qiu, G. Sterman, Phys. Lett. B 613, 45 (2005)

30. G.C. Nayak, J. Qiu, G. Sterman, Phys. Rev. D 72, 114012 (2005)

31. G.C. Nayak, J. Qiu, G. Sterman, Phys. Rev. D 74, 074007 (2006)

32. E. Braaten, S. Fleming, T.C. Tuan, Annu. Rev. Nucl. Part. Sci. 46, 197 (1996). arXiv:hep-ph/9602374

33. R. Tucci, Phys. Rev. D 32, 945 (1985)

34. G.C. Nayak, Ann. Phys. 325, 514 (2010)

35. See for example, T. Muta, Foundations of Quantum Chromodynamics, vol. 5. Lecture Notes in Physics (World Scientific, Singapore, 2009)

36. See for example, I. Stewart, The 19'th Taiwan Spring School on Particles and Fields, April 2006

37. L.F. Abbott, Nucl. Phys. B 185, 189 (1981)

38. J.C. Collins, D.E. Soper, Nucl. Phys. B 193, 381 (1981). Erratumibid.B213 (1983) 545

39. J.C. Collins, D.E. Soper, Nucl. Phys. B 194, 445 (1982)

40. J.C. Collins, D.E. Soper, G. Sterman, Nucl. Phys. B 261, 104 (1985)

41. C.W. Bauer, D. Pirjol, I.W. Stewart, Phys. Rev. D 65, 054022 (2002). arXiv:hep-ph/0109045

42. J. Chay, C. Kim, Y.G. Kim, J.-P. Lee, Phys. Rev. D 71, 056001 (2005). arXiv:hep-ph/0412110

43. C.F. Berger, arXiv:hep-ph/0305076

44. G.C. Nayak, Ann. Phys. 324, 2579 (2009)

45. G.C. Nayak, Ann. Phys. 325, 682 (2010)

46. G.T. Bodwin, Phys. Rev. D 31, 2616 (1985)
47. R. Frederix, Wilson lines in $Q C D$, nikhef/masters-thesis (2005)

48. J.C. Collins, D.E. Soper, G. Sterman, arXiv:hep-ph/0409313

49. G.C. Nayak, JHEP 1303, 001 (2013)

50. G.C. Nayak, Eur. Phys. J. C 73, 2442 (2013)

51. J. Schwinger, Phys. Rev. Lett. 3, 296 (1959)

52. J. Ye, J. Phys. Condens. Matter 16, 4465 (2004). arXiv:cond-mat/0206158

53. C.S. Lam, J. Math. Phys. 39, 5543 (1998)

54. P.M. Fishbane, S. Gasiorowwicz, P. Kaus, Phys. Rev. D 24, 2324 (1981)

55. C.W. Bauer, S. Fleming, M. Luke, Phys. Rev. D 63, 014006 (2001)

56. C.W. Bauer, S. Fleming, D. Pirjol, I.W. Stewart, Phys. Rev. D 63, $114020(2001)$

57. C.W. Bauer, I.W. Stewart, Phys. Lett. B 516, 134 (2001)

58. G.C. Nayak, J. Qiu, G. Sterman, Phys. Rev. Lett. 99, 212001 (2007)

59. G.C. Nayak, J. Qiu, G. Sterman, Phys. Rev. D 77, 034022 (2008)

60. G.C. Nayak, P. van Nieuwenhuizen, Phys. Rev. D 71, 125001 (2005)

61. G.C. Nayak, Phys. Rev. D 72, 125010 (2005)

62. G. Grammer, D.R. Yennie, Phys. Rev. D 8, 4332 (1973)

63. G. 't Hooft, Nucl. Phys. B 62, 444 (1973)

64. H. Klueberg-Stern, J.B. Zuber, Phys. Rev. D 12, 482 (1975)

65. H. Klueberg-Stern, J.B. Zuber, Phys. Rev. D 12, 3159 (1975)

66. J. Schwinger, Phys. Rev. 82, 664 (1951)

67. M.E. Peskin, D.V. Schroeder, Introduction to Quantum Field Theory (Perseus Books Publishing, L.L.C, Berkeley, California, 1995)

68. R.F. Dashen, D.J. Gross, Phys. Rev. D 23, 2340 (1981)

69. G.C. Nayak, Eur. Phys. J. C 64, 73 (2009). arXiv:0812.5054 [hep$\mathrm{ph}]$

70. F. Mandl, G. Shaw, Quantum Field Theory (Wiley, New York, 1984) 